\title{
SOME ELEMENTARY INEQUALITIES IN GAS DYNAMICS EQUATION
}

\section{A. KLYACHIN, A. V. KOCHETOV, AND V. M. MIKLYUKOV}

Received 12 January 2005; Accepted 25 August 2005

We describe the sets on which difference of solutions of the gas dynamics equation satisfy some special conditions. By virtue of nonlinearity of the equation the sets depend on the solution gradient quantity. We show double-ended estimates of the given sets and some properties of these estimates.

Copyright (C) 2006 V. A. Klyachin et al. This is an open access article distributed under the Creative Commons Attribution License, which permits unrestricted use, distribution, and reproduction in any medium, provided the original work is properly cited.

\section{Main results}

Consider the gas dynamics equation

$$
\sum_{c=1}^{n} \frac{\partial}{\partial x_{i}}\left(\sigma(|\nabla f|) f_{x_{i}}\right)=0
$$

where

$$
\sigma(t)=\left(1-\frac{\gamma-1}{2} t^{2}\right)^{1 /(\gamma-1)}
$$

Here $\gamma$ is a constant, $-\infty<\gamma<+\infty$. This equation describes the velocity potential of a steady-state flow of ideal gas in the adiabatic process. In the case $n=2$ the parameter $\gamma$ characterizes the flow of substance. For different values $\gamma$ it can be a flow of gas, fluid, plastic, electric or chemical field in different mediums, and so forth (see, e.g., [1, Section 2], [2, Section 15, Chapter IV]). For $\gamma=1 \pm 0$ we assume

$$
\sigma(t)=\exp \left\{-\frac{1}{2} t^{2}\right\}
$$


2 Some elementary inequalities in gas dynamics equation

The case of $\gamma=-1$ is known as the minimal surface equation (Chaplygin's gas):

$$
\operatorname{div}\left(\frac{\nabla f}{\sqrt{1+|\nabla f|^{2}}}\right)=0
$$

For $\gamma=-\infty,(1.1)$ becomes the Laplace equation.

In general, a solution of (1.1) with a function $\sigma$ of variables $\left(x_{1}, \ldots, x_{n}\right)$ is called $\sigma$ harmonic function. Such functions were studied in many works (see., e.g., [3, 4] and literature quoted therein).

We set $\Omega_{\gamma}=\mathbb{R}^{n}$ for $\gamma \leq 1$,

$$
\Omega_{\gamma}=\left\{\xi \in \mathbb{R}^{n}:|\xi|<\sqrt{\frac{2}{\gamma-1}}\right\} \text { for } \gamma>1
$$

The following inequalities were crucial in previous analysis of solutions to (1.1) for $\gamma=-1$ (see [5-9]):

$$
\begin{gathered}
c_{1} \sum_{i=1}^{n}\left(\xi_{i}-\eta_{i}\right)^{2} \leq \sum_{i=1}^{n}\left(\sigma(|\xi|) \xi_{i}-\sigma(|\eta|) \eta_{i}\right)\left(\xi_{i}-\eta_{i}\right), \quad \xi, \eta \in \Omega_{\gamma}, \\
\sum_{i=1}^{n}\left(\sigma(|\xi|) \xi_{i}-\sigma(|\eta|) \eta_{i}\right)^{2} \leq c_{2} \sum_{i=1}^{n}\left(\sigma(|\xi|) \xi_{i}-\sigma(|\eta|) \eta_{i}\right)\left(\xi_{i}-\eta_{i}\right), \quad \xi, \eta \in \Omega_{\gamma} .
\end{gathered}
$$

Here $\xi=\left(\xi_{1}, \xi_{2}, \ldots, \xi_{n}\right), \eta=\left(\eta_{1}, \eta_{2}, \ldots, \eta_{n}\right)$ and $c_{1}>0, c_{2}>0$ are constants not depending on $\xi$ and $\eta$.

In general, the latter inequalities are valid only on subsets of $\Omega_{\gamma} \times \Omega_{\gamma}$ with $c_{1}$ and $c_{2}$ depending on these subsets. The purpose of the present paper is to describe that dependence.

Introduce the sets

$$
\begin{aligned}
& \mathscr{A}_{\gamma}\left(c_{1}\right)=\left\{(\xi, \eta) \in \Omega_{\gamma} \times \Omega_{\gamma}: \xi, \eta \text { satisfy }(1.6)\right\} \\
& \mathscr{B}_{\gamma}\left(c_{2}\right)=\left\{(\xi, \eta) \in \Omega_{\gamma} \times \Omega_{\gamma}: \xi, \eta \text { satisfy }(1.7)\right\}
\end{aligned}
$$

Generally, the sets $\mathscr{A}_{\gamma}\left(c_{1}\right)$ and $\mathscr{B}_{\gamma}\left(c_{2}\right)$ have a complicated structure. We will describe them by comparing with canonical sets of the "simplest form."

We set $\Sigma_{\gamma}=\{x \in \mathbb{R}: x \geq 0\}$ for $\gamma \leq 1$ and

$$
\Sigma_{\gamma}=\left\{x \in \mathbb{R}: 0 \leq x<\sqrt{\frac{2}{\gamma-1}}\right\} \quad \text { for } \gamma>1
$$


V. A. Klyachin et al. 3

For every $\gamma \in \mathbb{R}$, define the functions $I_{\gamma}^{-}$and $I_{\gamma}^{+}$on $\Sigma_{\gamma} \times \Sigma_{\gamma}$ by

$$
\begin{aligned}
& I_{\gamma}^{-}(x, y)= \begin{cases}\frac{x \sigma(x)-y \sigma(y)}{x-y} & \text { if } x \neq y, \\
\sigma(x)+\sigma^{\prime}(x) x & \text { if } x=y,\end{cases} \\
& I_{\gamma}^{+}(x, y)= \begin{cases}\frac{x \sigma(x)+y \sigma(y)}{x+y} & \text { if } x^{2}+y^{2}>0, \\
1 & \text { if } x=y=0 .\end{cases}
\end{aligned}
$$

Note that the functions $I_{\gamma}^{-}$and $I_{\gamma}^{+}$are continuous on the closing of $\Sigma_{\gamma} \times \Sigma_{\gamma}$ and they are infinitely differentiable at each inner point of $\Sigma_{\gamma} \times \Sigma_{\gamma}$.

For arbitrary $\varepsilon \geq 0$ we put $W_{\gamma}^{-}(\varepsilon)=\left\{(\xi, \eta) \in \Omega_{\gamma} \times \Omega_{\gamma}: I_{\gamma}^{-}(|\xi|,|\eta|) \geq \varepsilon\right\}, W_{\gamma}^{+}(\varepsilon)=$ $\left\{(\xi, \eta) \in \Omega_{\gamma} \times \Omega_{\gamma}: I_{\gamma}^{+}(|\xi|,|\eta|) \geq \varepsilon\right\}, V_{\gamma}^{-}(\varepsilon)=\left\{(\xi, \eta) \in \Omega_{\gamma} \times \Omega_{\gamma}: I_{\gamma}^{-}(|\xi|,|\eta|) \leq \varepsilon\right\}, V_{\gamma}^{+}(\varepsilon)=$ $\left\{(\xi, \eta) \in \Omega_{\gamma} \times \Omega_{\gamma}: I_{\gamma}^{+}(|\xi|,|\eta|) \leq \varepsilon\right\}$.

Also we will need the sets $D_{\gamma}=\left\{(\xi, \xi) \in \Omega_{\gamma} \times \Omega_{\gamma}\right\}, Q_{\gamma}=\left\{(\xi, \eta) \in \Omega_{\gamma} \times \Omega_{\gamma}: \xi \sigma(|\xi|)=\right.$ $\eta \sigma(|\eta|)\}$.

The main result of our paper are the following theorems.

Theorem 1.1. For every $\gamma \in \mathbb{R}$,

$$
\begin{gathered}
\left(W_{\gamma}^{-}(\varepsilon) \cup D_{\gamma}\right) \subset \mathscr{A}_{\gamma}(\varepsilon) \subset\left(W_{\gamma}^{+}(\varepsilon) \cup D_{\gamma}\right) \quad \forall \varepsilon \in(0,1), \\
\mathscr{A}_{\gamma}(\varepsilon)=D_{\gamma} \quad \forall \varepsilon \in[1,+\infty) .
\end{gathered}
$$

Theorem 1.2. (a) If $\gamma \in(-\infty,-1]$, then

$$
\begin{gathered}
\left(V_{\gamma}^{+}(\varepsilon) \cup D_{\gamma}\right) \subset \mathscr{B}_{\gamma}(\varepsilon) \subset\left(V_{\gamma}^{-}(\varepsilon) \cup D_{\gamma}\right) \quad \forall \epsilon \in(0,1), \\
\mathscr{B}_{\gamma}(\varepsilon)=\mathbb{R}^{2 n} \quad \forall \varepsilon \in[1,+\infty) .
\end{gathered}
$$

(b) If $\gamma \in(-1,+\infty)$, then

$$
\begin{gathered}
\left(V_{\gamma}^{+}(\varepsilon) \cap W_{\gamma}^{-}(0)\right) \subset \mathscr{B}_{\gamma}(\varepsilon) \subset\left(V_{\gamma}^{-}(\varepsilon) \cup Q_{\gamma}\right) \quad \forall \varepsilon \in(0,1), \\
W_{\gamma}^{-}(0) \subset \mathscr{B}_{\gamma}(\varepsilon) \quad \forall \varepsilon \in[1,+\infty) .
\end{gathered}
$$

Relation (1.14) was first proved for $\gamma=-1$ and $\varepsilon=1$ in [5] and later repeatedly in [6-9].

\section{Properties of $\sigma$}

Consider the equation

$$
\theta^{\prime}(t)=\varepsilon,
$$


4 Some elementary inequalities in gas dynamics equation

where $\theta(t)=t \sigma(t)$ and $\varepsilon$ is an arbitrary parameter. It is easy to verify that for $\gamma \neq 1,(2.1)$ can be rewritten in the following form:

$$
\frac{2}{\gamma-1} \sigma^{2-\gamma}(t)-\frac{\gamma+1}{\gamma-1} \sigma(t)+\varepsilon=0
$$

For arbitrary $\varepsilon \in(0,1)$ we set

$$
\begin{gathered}
r_{\gamma}(\varepsilon)=\sqrt{\frac{2\left(1-\varepsilon^{\gamma-1}\right)}{\gamma-1}} \text { if } \gamma \neq 1, \\
r_{1}(\varepsilon)=\sqrt{-2 \ln \varepsilon} .
\end{gathered}
$$

Observe that $r_{\gamma}(\varepsilon) \in \Sigma_{\gamma}$ for every $\gamma \in \mathbb{R}$ and every $\varepsilon \in(0,1)$.

The following assertions hold.

(1) Let $\gamma \in \mathbb{R}$. Then the domain of $\sigma$ is the set $\Sigma_{\gamma}$. Moreover, $\sigma(0)=1, \sigma(+\infty)=0$ for $\gamma \leq 1$ and $\sigma(\sqrt{2 /(\gamma-1)})=0$ for $\gamma>1$.

(2) For each $\gamma \in \mathbb{R}$ we have

$$
0<\sigma(t) \leq 1 \quad \forall t \in \Sigma_{\gamma}
$$

(3) Let $\gamma \in \mathbb{R}$. Then $\sigma^{\prime}(0)=0$ and

$$
\sigma^{\prime}(t)<0 \quad \forall t>0, t \in \Sigma_{\gamma}
$$

(4) If $\gamma \in(-\infty,-1]$, then

$$
\begin{aligned}
& \theta^{\prime}(0)=1, \quad \theta^{\prime}(+\infty)=0, \\
& \theta^{\prime}(t)>0 \quad \forall t \in[0,+\infty) .
\end{aligned}
$$

(5) If $\gamma \in(-1,+\infty)$, then

$$
\begin{aligned}
& \theta^{\prime}(0)=1, \quad \theta^{\prime}\left(\sqrt{\frac{2}{\gamma+1}}\right)=0, \\
& \theta^{\prime}(t)>0 \quad \forall t \in\left(0, \sqrt{\frac{2}{\gamma+1}}\right), \\
& \theta^{\prime}(t)<0 \quad \forall t>\sqrt{\frac{2}{\gamma+1}}, t \in \Sigma_{\gamma} .
\end{aligned}
$$


Moreover,

$$
\begin{gathered}
\theta^{\prime}(+\infty)=0 \quad \text { if } \gamma \in(-1,1], \\
\theta^{\prime}\left(\sqrt{\frac{2}{\gamma-1}}\right)=0 \quad \text { if } \gamma \in(1,2), \\
\theta^{\prime}\left(\sqrt{\frac{2}{\gamma-1}}\right)=-2 \quad \text { if } \gamma=2, \\
\theta^{\prime}\left(\sqrt{\frac{2}{\gamma-1}}-0\right)=-\infty \quad \text { if } \gamma \in(2,+\infty) .
\end{gathered}
$$

(6) If $\gamma \in(-\infty,-1] \cup[2,+\infty)$, then $\theta^{\prime \prime}(0)=0$ and

$$
\theta^{\prime \prime}(t)<0 \quad \forall t>0, t \in \Sigma_{\gamma} .
$$

(7) If $\gamma \in(-1,2)$, then

$$
\begin{aligned}
& \theta^{\prime \prime}(0)=0, \quad \theta^{\prime \prime}\left(\sqrt{\frac{6}{\gamma+1}}\right)=0, \\
& \theta^{\prime \prime}(t)<0 \quad \forall t \in\left(0, \sqrt{\frac{6}{\gamma+1}}\right), \\
& \theta^{\prime \prime}(t)>0 \quad \forall t>\sqrt{\frac{6}{\gamma+1}}, t \in \Sigma_{\gamma} .
\end{aligned}
$$

(8) For every $\gamma \in \mathbb{R}$ and every $\epsilon \in(0,1),(2.1)$ has a unique positive solution $s_{\gamma}(\varepsilon) \in$ $\left(0, r_{\gamma}(\varepsilon)\right)$ and

$$
\theta^{\prime}(t)>\varepsilon \quad \forall t \in\left[0, s_{\gamma}(\varepsilon)\right), \quad \theta^{\prime}(t)<\varepsilon \quad \forall t>s_{\gamma}(\varepsilon), t \in \Sigma_{\gamma} .
$$

Moreover, for every $\gamma>-1$ and $\epsilon \in(0,1)$,

$$
s_{\gamma}(\varepsilon)<\sqrt{\frac{2}{\gamma+1}} .
$$

(9) Let $\gamma \in \mathbb{R}$. Then for all $x, y \in \Sigma_{\gamma}, x^{2}+y^{2}>0$,

$$
I_{\gamma}^{-}(x, y) \leq I_{\gamma}^{+}(x, y)<1 .
$$


6 Some elementary inequalities in gas dynamics equation

Proof. The proof of assertions (1)-(7) follows from the equalities

$$
\begin{aligned}
& \sigma^{\prime}(t)= \begin{cases}-t\left(1-\frac{\gamma-1}{2} t^{2}\right)^{(2-\gamma) /(\gamma-1)} & \text { if } \gamma \neq 1, \\
-t \exp \left\{-\frac{1}{2} t^{2}\right\} & \text { if } \gamma=1,\end{cases} \\
& \theta^{\prime}(t)= \begin{cases}\left(1-\frac{\gamma+1}{2} t^{2}\right)\left(1-\frac{\gamma-1}{2} t^{2}\right)^{(2-\gamma) /(\gamma-1)} & \text { if } \gamma \neq 1, \\
\left(1-t^{2}\right) \exp \left\{-\frac{1}{2} t^{2}\right\} & \text { if } \gamma=1,\end{cases} \\
& \theta^{\prime \prime}(t)= \begin{cases}-t\left(3-\frac{\gamma+1}{2} t^{2}\right)\left(1-\frac{\gamma-1}{2} t^{2}\right)^{(3-2 \gamma) /(\gamma-1)} & \text { if } \gamma \neq 1, \\
t\left(t^{2}-3\right) \exp \left\{-\frac{1}{2} t^{2}\right\} & \text { if } \gamma=1 .\end{cases}
\end{aligned}
$$

Let $\gamma \in \mathbb{R}$ and $\epsilon \in(0,1)$. Suppose that $s_{\gamma}(\varepsilon) \in \Sigma_{\gamma}$ satisfies (2.1). We have

$$
\sigma\left(r_{\gamma}(\varepsilon)\right)=\varepsilon=\theta^{\prime}\left(s_{\gamma}(\varepsilon)\right)=\sigma\left(s_{\gamma}(\varepsilon)\right)+s_{\gamma}(\varepsilon) \sigma^{\prime}\left(s_{\gamma}(\varepsilon)\right)<\sigma\left(s_{\gamma}(\varepsilon)\right) .
$$

From this $s_{\gamma}(\varepsilon)<r_{\gamma}(\varepsilon)$. Next, using assertions (4)-(7), we obtain assertion (8).

We prove assertion (9). Let $x, y \in \Sigma_{\gamma}, x^{2}+y^{2}>0$. If $x=y$, then

$$
I_{\gamma}^{-}(x, y)=\sigma(x)+x \sigma^{\prime}(x)<\sigma(x)=I_{\gamma}^{+}(x, y)<1 .
$$

Suppose that $x>y$. Since

$$
\sigma(x)<\sigma(y)
$$

we obtain

$$
\begin{aligned}
I_{\gamma}^{-}(x, y) & =\frac{x \sigma(x)-y \sigma(y)}{x-y} \leq \frac{x \sigma(x)-y \sigma(x)}{x-y}=\sigma(x) \\
& =\frac{x \sigma(x)+y \sigma(x)}{x+y} \leq \frac{x \sigma(x)+y \sigma(y)}{x+y}=I_{\gamma}^{+}(x, y) \\
& <\frac{x \sigma(y)+y \sigma(y)}{x+y}=\sigma(y) \leq 1 .
\end{aligned}
$$

The case $x<y$ is analogous.

\section{Properties of $W_{\gamma}^{-}(\varepsilon), W_{\gamma}^{+}(\varepsilon), V_{\gamma}^{-}(\varepsilon)$, and $V_{\gamma}^{+}(\varepsilon)$}

Here we study the sets $W_{\gamma}^{-}(\varepsilon), W_{\gamma}^{+}(\varepsilon), V_{\gamma}^{-}(\varepsilon)$ and $V_{\gamma}^{+}(\varepsilon)$.

We say that a set $G \subset \mathbf{R}^{n}$ is linearly connected if any pair of points $x, y \in G$ can be joined on $D$ by an arc. 
The following assertions hold.

(1) $W_{\gamma}^{-}(\varepsilon)=W_{\gamma}^{+}(\varepsilon)=\varnothing$ for every $\gamma \in \mathbb{R}$ and $\varepsilon>1$.

(2) $W_{\gamma}^{-}(1)=W_{\gamma}^{+}(1)=\{0\}$ for every $\gamma \in \mathbb{R}$.

(3) $W_{\gamma}^{-}(0)=\mathbb{R}^{4}$ for every $\gamma \leq-1$.

(4) $W_{\gamma}^{+}(0)=\Omega_{\gamma} \times \Omega_{\gamma}$ for every $\gamma \in \mathbb{R}$.

(5) $W_{\gamma}^{-}(\varepsilon) \subset W_{\gamma}^{+}(\varepsilon)$ for every $\gamma \in \mathbb{R}$ and $\varepsilon \in(0,1)$.

(6) $V_{\gamma}^{-}(\varepsilon)=V_{\gamma}^{+}(\varepsilon)=\Omega_{\gamma} \times \Omega_{\gamma}$ for every $\gamma \in \mathbb{R}$ and $\varepsilon \geq 1$.

(7) $V_{\gamma}^{-}(0)=\varnothing$ for every $\gamma \leq-1$.

(8) $V_{\gamma}^{+}(0)=\varnothing$ for every $\gamma \in \mathbb{R}$.

(9) $V_{\gamma}^{+}(\varepsilon) \subset V_{\gamma}^{-}(\varepsilon)$ for every $\gamma \in \mathbb{R}$ and $\varepsilon \in(0,1)$.

(10) The set $W_{\gamma}^{-}(\varepsilon)$ is linearly connected for every $\gamma \in \mathbb{R}$ and $\varepsilon \in(0,1)$.

(11) The set $W_{\gamma}^{-}(0)$ is linearly connected for every $\gamma>-1$.

(12) The set $W_{\gamma}^{+}(\varepsilon)$ is linearly connected for every $\gamma \in \mathbb{R}$ and $\varepsilon \in(0,1)$.

(13) For every $\gamma \in \mathbb{R}$ and $\varepsilon \in(0,1)$, we have

$$
\left\{(\xi, \eta) \in \Omega_{\gamma} \times \Omega_{\gamma}:|\xi| \leq s_{\gamma}(\varepsilon),|\eta| \leq s_{\gamma}(\varepsilon)\right\} \subset W_{\gamma}^{-}(\varepsilon) .
$$

Here $s_{\gamma}(\varepsilon)$ is a unique positive solution of (2.1).

(14) For every $\gamma \in \mathbb{R}$ and $\varepsilon \in(0,1)$, we have

$$
W_{\gamma}^{-}(\varepsilon) \subset\left\{(\xi, \eta) \in \Omega_{\gamma} \times \Omega_{\gamma}:|\xi| \leq r_{\gamma}(\varepsilon),|\eta| \leq r_{\gamma}(\varepsilon)\right\} .
$$

(15) If $\gamma>-1$, then

$$
\left\{(\xi, \eta) \in \Omega_{\gamma} \times \Omega_{\gamma}:|\xi| \leq \sqrt{\frac{2}{\gamma+1}},|\eta| \leq \sqrt{\frac{2}{\gamma+1}}\right\} \subset W_{\gamma}^{-}(0) .
$$

(16) For every $\gamma \in \mathbb{R}$ and $\varepsilon \in(0,1)$, we have

$$
\left\{(\xi, \eta) \in \Omega_{\gamma} \times \Omega_{\gamma}:|\xi| \leq r_{\gamma}(\varepsilon),|\eta| \leq r_{\gamma}(\varepsilon)\right\} \subset W_{\gamma}^{+}(\varepsilon) .
$$

(17) For every $\gamma \in \mathbb{R}$ and $\varepsilon \in(0,1)$, we have

$$
V_{\gamma}^{-}(\varepsilon) \subset\left\{(\xi, \eta) \in \Omega_{\gamma} \times \Omega_{\gamma}:|\xi| \geq s_{\gamma}(\varepsilon) \text { or }|\eta| \geq s_{\gamma}(\varepsilon)\right\} .
$$

(18) For every $\gamma \in \mathbb{R}$ and $\varepsilon \in(0,1)$, we have

$$
\left\{(\xi, \eta) \in \Omega_{\gamma} \times \Omega_{\gamma}:|\xi| \geq r_{\gamma}(\varepsilon) \text { or }|\eta| \geq r_{\gamma}(\varepsilon)\right\} \subset V_{\gamma}^{-}(\varepsilon) .
$$

(19) If $\gamma>-1$, then

$$
V_{\gamma}^{-}(0) \subset\left\{(\xi, \eta) \in \Omega_{\gamma} \times \Omega_{\gamma}:|\xi| \geq \sqrt{\frac{2}{\gamma+1}} \text { or }|\eta| \geq \sqrt{\frac{2}{\gamma+1}}\right\} .
$$

(20) For every $\gamma \in \mathbb{R}$ and $\varepsilon \in(0,1)$, we have

$$
V_{\gamma}^{+}(\varepsilon) \subset\left\{(\xi, \eta) \in \Omega_{\gamma} \times \Omega_{\gamma}:|\xi| \geq r_{\gamma}(\varepsilon) \text { or }|\eta| \geq r_{\gamma}(\varepsilon)\right\} .
$$


8 Some elementary inequalities in gas dynamics equation

Proof of assertions (1)-(9). The proof follows from assertions (4) and (9) of Section 2.

Proof of assertions (10)-(12). We prove assertion (10). Fix $\gamma \in \mathbb{R}, \varepsilon \in(0,1)$, and a nonzero point $\zeta=(\xi, \eta) \in W_{\gamma}^{-}(\varepsilon)$. To prove the statement, it is sufficient to show that $W_{\gamma}^{-}(\varepsilon)$ contains the segment $\mathscr{L}=\{(\xi t, \eta t): 0 \leq t \leq 1\}$ with the endpoints 0 and $\zeta$.

Indeed, let $\zeta^{\prime}, \zeta^{\prime \prime} \in W_{\gamma}^{-}(\varepsilon)$ be arbitrary. Let $\mathscr{L}^{\prime}, \mathscr{L}^{\prime \prime}$ be the segments with the endpoints $0, \zeta^{\prime}$ and $0, \zeta^{\prime \prime}$, respectively. Denote by $\mathscr{L}^{\prime} \cup \mathscr{L}^{\prime \prime}$ the double curve which consists of two segments $\mathscr{L}^{\prime}$ and $\mathscr{L}^{\prime \prime}$. Then this double curve will join the points $\zeta^{\prime}, \zeta^{\prime \prime}$ and it will lie on $W_{\gamma}^{-}(\varepsilon)$.

Assume that $I_{\gamma}(x, y) \geq \epsilon$. As above, for the case $x>y$ we obtain

$$
\varepsilon \leq I_{\gamma}^{-}(x, y) \leq \sigma(x)<\sigma(y) .
$$

From this $x, y \in\left[0, r_{\gamma}(\varepsilon)\right]$. The case $x<y$ is analogous. Suppose that $x=y$. Then

$$
\varepsilon \leq I_{\gamma}^{-}(x, y)=\theta^{\prime}(x)=\sigma(x)+x \sigma^{\prime}(x) \leq \sigma(x)=\sigma(y),
$$

and consequently $x, y \in\left[0, r_{\gamma}(\varepsilon)\right]$. Thus if $I_{\gamma}(x, y) \geq \epsilon$, then $x, y \in\left[0, r_{\gamma}(\varepsilon)\right]$.

Further we will need the function

$$
\mu(x)=x(\sigma(x)-\varepsilon)
$$

It is easy to see that for all $x, y \in\left[0, r_{\gamma}(\varepsilon)\right], x \neq y$,

$$
I_{\gamma}^{-}(x, y)=\varepsilon \Longleftrightarrow \mu(x)=\mu(y) .
$$

Define the monotonicity intervals of $\mu$. Since

$$
\mu^{\prime}(x)=\theta^{\prime}(x)-\varepsilon
$$

from assertion (8) of Section 2 it follows that the function $\mu$ is strictly increasing on $\left[0, s_{\gamma}(\varepsilon)\right]$ and strictly decreasing on $\left[s_{\gamma}(\varepsilon), r_{\gamma}(\varepsilon)\right]$. Moreover,

$$
\mu(0)=\mu\left(r_{\gamma}(\varepsilon)\right)=0 .
$$

Note that if $I_{\gamma}^{-}(x, y)=\varepsilon$ and $x=y$, then $x=y=s_{\gamma}(\varepsilon)$. Consequently for each $x \in$ $\left[0, r_{\gamma}(\varepsilon)\right]$ there is a unique number $y \in\left[0, r_{\gamma}(\varepsilon)\right]$, satisfying (3.12). Therefore there exists the function $g:\left[0, r_{\gamma}(\varepsilon)\right] \rightarrow\left[0, r_{\gamma}(\varepsilon)\right]$ such that for all $x, y \in\left[0, r_{\gamma}(\varepsilon)\right]$,

$$
I_{\gamma}^{-}(x, y)=\varepsilon \Longleftrightarrow y=g(x) .
$$

In addition

$$
\begin{aligned}
& s_{\gamma}(\varepsilon)<g(x) \leq r_{\gamma}(\varepsilon) \quad \text { if } x \in\left[0, s_{\gamma}(\varepsilon)\right), \\
& 0 \leq g(x)<s_{\gamma}(\varepsilon) \quad \text { if } x \in\left(s_{\gamma}(\varepsilon), r_{\gamma}(\varepsilon)\right],
\end{aligned}
$$


as well as

$$
g(0)=r_{\gamma}(\varepsilon), \quad g\left(s_{\gamma}(\varepsilon)\right)=s_{\gamma}(\varepsilon), \quad g\left(r_{\gamma}(\varepsilon)\right)=0 .
$$

Note that the function $I_{\gamma}^{-}(x, y)$ is infinitely differentiable at each point of $\left[0, r_{\gamma}(\varepsilon)\right] \times$ $\left[0, r_{\gamma}(\varepsilon)\right]$. Fix arbitrary $x_{0}, y_{0} \in\left[0, r_{\gamma}(\varepsilon)\right], x_{0} \neq y_{0}$, satisfying (3.15). We have

$$
\begin{gathered}
\frac{\partial}{\partial x} I_{\gamma}^{-}\left(x_{0}, y_{0}\right)=\frac{\theta^{\prime}\left(x_{0}\right)\left(x_{0}-y_{0}\right)-\left(\theta\left(x_{0}\right)-\theta\left(y_{0}\right)\right)}{\left(x_{0}-y_{0}\right)^{2}}=\frac{\theta^{\prime}\left(x_{0}\right)-\varepsilon}{x_{0}-y_{0}} \neq 0, \\
\frac{\partial}{\partial y} I_{\gamma}^{-}\left(x_{0}, y_{0}\right)=\frac{\theta^{\prime}\left(y_{0}\right)\left(y_{0}-x_{0}\right)-\left(\theta\left(y_{0}\right)-\theta\left(x_{0}\right)\right)}{\left(y_{0}-x_{0}\right)^{2}}=\frac{\theta^{\prime}\left(y_{0}\right)-\varepsilon}{y_{0}-x_{0}} \neq 0 .
\end{gathered}
$$

Using the implicit function theorem, we obtain

$$
g^{\prime}\left(x_{0}\right)=-\left[\frac{\partial}{\partial y} I_{\gamma}^{-}\left(x_{0}, g\left(x_{0}\right)\right)\right]^{-1}\left[\frac{\partial}{\partial x} I_{\gamma}^{-}\left(x_{0}, g\left(x_{0}\right)\right)\right]=\frac{\theta^{\prime}\left(x_{0}\right)-\varepsilon}{\theta^{\prime}\left(g\left(x_{0}\right)\right)-\varepsilon} .
$$

By assertion (8) of Section 2, (3.16), it follows that

$$
g^{\prime}\left(x_{0}\right)<0 .
$$

Thus the function $y=g(x)$ is strictly decreasing on $\left[0, r_{\gamma}(\varepsilon)\right]$.

We prove that the segment $\mathscr{L}$ lies in $W_{\gamma}^{-}(\varepsilon)$.

Indeed, assume that $|\xi| \leq|\eta|$ and for some $t \in(0,1)$,

$$
I_{\gamma}^{-}(|\xi t|,|\eta t|)<\varepsilon
$$

Then there is a number $t_{0} \in(0,1)$ such that

$$
I_{\gamma}^{-}\left(\left|\xi t_{0}\right|,\left|\eta t_{0}\right|\right)=\varepsilon
$$

and hence $\left|\eta t_{0}\right|=g\left(\left|\xi t_{0}\right|\right)$.

Since $0<r_{\gamma}(\varepsilon)=g(0)$ and $I_{\gamma}^{-}(0,0)=1>\varepsilon$, we have $|\eta| \leq g(|\xi|)$. We deduce

$$
\frac{g(|\xi|)}{t_{0}} \leq \frac{g\left(\left|\xi t_{0}\right|\right)}{t_{0}}=|\eta| \leq g(|\xi|) .
$$

From this $t_{0} \geq 1$ and we arrive at a contradiction. The case $|\xi|>|\eta|$ is analogous. Thus $W_{\gamma}^{-}(\varepsilon)$ contains $\mathscr{L}$.

The proof of assertion (11) is analogous.

Now we prove assertion (12). We fix $\gamma \in \mathbb{R}, \varepsilon \in(0,1)$, and a nonzero point $\zeta=(\xi, \eta) \in$ $W_{\gamma}^{+}(\varepsilon)$. As above, to prove this statement, it is sufficient to show that $W_{\gamma}^{+}(\varepsilon)$ contains the segment $\mathscr{L}$. We have

$$
I_{\gamma}^{+}(|\xi t|,|\eta t|)=\frac{|\xi| \sigma(|\xi t|)+|\eta| \sigma(|\eta t|)}{|\xi|+|\eta|}>\frac{|\xi| \sigma(|\xi|)+|\eta| \sigma(|\eta|)}{|\xi|+|\eta|} \geq \varepsilon
$$

for all $t \in(0,1)$. Thus $W_{\gamma}^{+}(\varepsilon)$ contains $\mathscr{L}$. 
10 Some elementary inequalities in gas dynamics equation

Proof of assertions (13), (15), (17), and (19). Let

$$
(\xi, \eta) \in\left\{(\xi, \eta) \in \Omega_{\gamma} \times \Omega_{\gamma}:|\xi| \leq s_{\gamma}(\varepsilon),|\eta| \leq s_{\gamma}(\varepsilon)\right\} .
$$

By assertion (8) of Section 2 it follows that

$$
\theta^{\prime}(|\xi|) \geq \varepsilon, \quad \theta^{\prime}(|\eta|) \geq \varepsilon .
$$

Suppose that $|\xi|=|\eta|$. We have

$$
I_{\gamma}^{-}(|\xi|,|\eta|)=\theta^{\prime}(|\xi|)=\theta^{\prime}(|\eta|) \geq \varepsilon .
$$

From this $(\xi, \eta) \in W_{\gamma}^{-}(\varepsilon)$.

Assume that $|\xi|<|\eta|$. Using the well-known Lagrange mean value theorem, we obtain

$$
I_{\gamma}^{-}(|\xi|,|\eta|)=\theta^{\prime}(c), \quad|\xi|<c<|\eta| .
$$

By assertion (8) of Section 2,

$$
\theta^{\prime}(c)>\varepsilon
$$

Therefore $(\xi, \eta) \in W_{\gamma}^{-}(\varepsilon)$. The case $|\xi|>|\eta|$ is analogous.

The proof of assertion (15) is analogous. Assertion (17) follows from assertion (13), and assertion (19) follows from assertion (15).

Proof of assertions (14) and (18). Let $(\xi, \eta) \in W_{\gamma}^{-}(\varepsilon)$. Assume that $|\xi|=|\eta|$. We have

$$
\varepsilon \leq I_{\gamma}^{-}(|\xi|,|\eta|)=\theta^{\prime}(|\xi|)=\sigma(|\xi|)+|\xi| \sigma^{\prime}(|\xi|) \leq \sigma(|\xi|)=\sigma(|\eta|) .
$$

Then the inequalities

$$
\sigma(|\xi|)=\sigma(|\eta|) \geq \varepsilon
$$

imply

$$
|\xi|=|\eta| \leq r_{\gamma}(\varepsilon)
$$

Hence

$$
(\xi, \eta) \in\left\{(\xi, \eta) \in \Omega_{\gamma} \times \Omega_{\gamma}:|\xi| \leq r_{\gamma}(\varepsilon),|\eta| \leq r_{\gamma}(\varepsilon)\right\}
$$

Now we assume that $|\xi|>|\eta|$. We have

$$
\varepsilon \leq I_{\gamma}^{-}(|\xi|,|\eta|)=\frac{|\xi| \sigma(|\xi|)-|\eta| \sigma(|\eta|)}{|\xi|-|\eta|} \leq \frac{|\xi| \sigma(|\xi|)-|\eta| \sigma(|\xi|)}{|\xi|-|\eta|}=\sigma(|\xi|)<\sigma(|\eta|) .
$$

From this

$$
(\xi, \eta) \in\left\{(\xi, \eta) \in \Omega_{\gamma} \times \Omega_{\gamma}:|\xi| \leq r_{\gamma}(\varepsilon),|\eta| \leq r_{\gamma}(\varepsilon)\right\}
$$


The case $|\xi|<|\eta|$ is analogous.

Assertion (18) follows from assertion (14).

Proof of assertions (16) and (20). Let

$$
(\xi, \eta) \in\left\{(\xi, \eta) \in \Omega_{\gamma} \times \Omega_{\gamma}:|\xi| \leq r_{\gamma}(\varepsilon),|\eta| \leq r_{\gamma}(\varepsilon)\right\} .
$$

Then

$$
\sigma(|\xi|) \geq \varepsilon, \quad \sigma(|\eta|) \geq \varepsilon
$$

Suppose $|\xi|=|\eta|$. Then

$$
I_{\gamma}^{+}(|\xi|,|\eta|)=\sigma(|\xi|) \geq \varepsilon
$$

Hence $(\xi, \eta) \in W_{\gamma}^{+}(\varepsilon)$.

Assume that $|\xi|>|\eta|$. We have

$$
I_{\gamma}^{+}(|\xi|,|\eta|)=\frac{|\xi| \sigma(|\xi|)+|\eta| \sigma(|\eta|)}{|\xi|+|\eta|} \geq \frac{|\xi| \sigma(|\xi|)+|\eta| \sigma(|\xi|)}{|\xi|+|\eta|}=\sigma(|\xi|) \geq \varepsilon
$$

From this $(\xi, \eta) \in W_{\gamma}^{+}(\varepsilon)$. The case $|\xi|<|\eta|$ is analogous.

Assertion (20) follows from assertion (16).

\section{Proofs of main theorems}

Introduce the sets $H_{\gamma}=\left\{(\xi, \eta) \in \Omega_{\gamma} \times \Omega_{\gamma}:|\xi|=|\eta|, \xi \neq \eta\right\}, G_{\gamma}=\left\{(\xi, \eta) \in \Omega_{\gamma} \times \Omega_{\gamma}\right.$ : $|\xi| \neq|\eta|\}, U_{\gamma}^{-}=\left\{(\xi, \eta) \in \Omega_{\gamma} \times \Omega_{\gamma}: I_{\gamma}^{-}(|\xi|,|\eta|)<0\right\}, U_{\gamma}^{+}=\left\{(\xi, \eta) \in \Omega_{\gamma} \times \Omega_{\gamma}: I_{\gamma}^{-}\right.$ $(|\xi|,|\eta|)>0\}, P_{\gamma}=\left\{(\xi, \eta) \in \Omega_{\gamma} \times \Omega_{\gamma}:|\xi| \sigma(|\xi|)=|\eta| \sigma(|\eta|), \quad \xi \sigma(|\xi|) \neq \eta \sigma(|\eta|)\right\}$, $F_{\gamma}^{+}(\varepsilon)=\left(V_{\gamma}^{+}(\varepsilon) \cap U_{\gamma}^{+}\right) \cup Q_{\gamma} \cup\left(V_{\gamma}^{+}(\varepsilon) \cap P_{\gamma}\right), F_{\gamma}^{-}(\varepsilon)=\left(V_{\gamma}^{-}(\varepsilon) \cap U_{\gamma}^{+}\right) \cup Q_{\gamma} \cup\left(V_{\gamma}^{+}(\varepsilon) \cap P_{\gamma}\right)$ $\cup\left(V_{\gamma}^{+}(\varepsilon) \cap U_{\gamma}^{-}\right)$.

For any $\xi, \eta \in \mathbb{R}^{n}$, their inner product is denoted by $\langle\xi, \eta\rangle$. Obviously inequalities (1.6) and (1.7) with some constant $\varepsilon>0$ can be written as

$$
\begin{gathered}
\varepsilon|\xi-\eta|^{2} \leq\langle\sigma(|\xi|) \xi-\sigma(|\eta|) \eta, \xi-\eta\rangle, \\
|\sigma(|\xi|) \xi-\sigma(|\eta|) \eta|^{2} \leq \varepsilon\langle\sigma(|\xi|) \xi-\sigma(|\eta|) \eta, \xi-\eta\rangle,
\end{gathered}
$$

respectively. Let $\varphi$ be the angle between the vectors $\xi$ and $\eta$. Then

$$
\begin{aligned}
|\xi-\eta|^{2} & =|\xi|^{2}+|\eta|^{2}-2|\xi||\eta| \cos \varphi, \\
\langle\sigma(|\xi|) \xi-\sigma(|\eta|) \eta, \xi-\eta\rangle & =\sigma(|\xi|)|\xi|^{2}+\sigma(|\eta|)|\eta|^{2}-(\sigma(|\xi|)+\sigma(|\eta|))|\xi||\eta| \cos \varphi, \\
|\sigma(|\xi|) \xi-\sigma(|\eta|) \eta|^{2} & =\sigma^{2}(|\xi|)|\xi|^{2}+\sigma^{2}(|\eta|)|\eta|^{2}-2 \sigma(|\xi|) \sigma(|\eta|)|\xi||\eta| \cos \varphi .
\end{aligned}
$$


12 Some elementary inequalities in gas dynamics equation

We set

$$
\begin{gathered}
\Upsilon(\varphi)=|\xi|^{2}+|\eta|^{2}-2|\xi||\eta| \cos \varphi \\
\Phi(\varphi)=\sigma(|\xi|)|\xi|^{2}+\sigma(|\eta|)|\eta|^{2}-(\sigma(|\xi|)+\sigma(|\eta|))|\xi||\eta| \cos \varphi \\
\Psi(\varphi)=\sigma^{2}(|\xi|)|\xi|^{2}+\sigma^{2}(|\eta|)|\eta|^{2}-2 \sigma(|\xi|) \sigma(|\eta|)|\xi||\eta| \cos \varphi .
\end{gathered}
$$

Proof of Theorem 1.1. Fix $\gamma \in \mathbb{R}$ and $\varepsilon>0$. It is clear that inequality (4.1) holds for all $(\xi, \eta) \in D_{\gamma}$.

Let $(\xi, \eta) \in \mathscr{A}_{\gamma}(\varepsilon) \cap H_{\gamma}$. In this case inequality (4.1) is rewritten in the form

$$
\varepsilon \leq \sigma(|\xi|)=\sigma(|\eta|)
$$

Obviously

$$
\mathscr{A}_{\gamma}(\epsilon) \cap H_{\gamma}=W_{\gamma}^{+}(\epsilon) \cap H_{\gamma}
$$

Using assertion (5) of Section 3, we see that

$$
\left(W_{\gamma}^{-}(\varepsilon) \cap H_{\gamma}\right) \subset\left(\mathscr{A}_{\gamma}(\varepsilon) \cap H_{\gamma}\right) \subset\left(W_{\gamma}^{+}(\varepsilon) \cap H_{\gamma}\right) .
$$

Let $(\xi, \eta) \in G_{\gamma}$. Then $\Upsilon(\varphi)>0$ and after simple calculations we find

$$
\frac{\partial}{\partial \varphi}\left(\frac{\Phi(\varphi)}{\Upsilon(\varphi)}\right)=\frac{(\sigma(|\eta|)-\sigma(|\xi|))\left(|\xi|^{2}-|\eta|^{2}\right)|\xi||\eta| \sin \varphi}{\Upsilon^{2}(\varphi)} .
$$

It is clear that

$$
(\sigma(|\eta|)-\sigma(|\xi|))\left(|\xi|^{2}-|\eta|^{2}\right)>0 .
$$

Therefore

$$
\begin{aligned}
\min _{\varphi \in[0, \pi]}\left(\frac{\Phi(\varphi)}{\Upsilon(\varphi)}\right) & =\frac{\Phi(0)}{\Upsilon(0)} \\
& =\frac{\sigma(|\xi|)|\xi|^{2}+\sigma(|\eta|)|\eta|^{2}-(\sigma(|\xi|)+\sigma(|\eta|))|\xi||\eta|}{(|\xi|-|\eta|)^{2}}=I_{\gamma}^{-}(|\xi|,|\eta|) \\
\max _{\varphi \in[0, \pi]}\left(\frac{\Phi(\varphi)}{\Upsilon(\varphi)}\right) & =\frac{\Phi(\pi)}{\Upsilon(\pi)} \\
& =\frac{\sigma(|\xi|)|\xi|^{2}+\sigma(|\eta|)|\eta|^{2}+(\sigma(|\xi|)+\sigma(|\eta|))|\xi||\eta|}{(|\xi|+|\eta|)^{2}}=I_{\gamma}^{+}(|\xi|,|\eta|) .
\end{aligned}
$$

Thus for all $(\xi, \eta) \in G_{\gamma}$,

$$
I_{\gamma}^{-}(|\xi|,|\eta|) \leq \frac{\langle\sigma(|\xi|) \xi-\sigma(|\eta|) \eta, \xi-\eta\rangle}{|\xi-\eta|^{2}} \leq I_{\gamma}^{+}(|\xi|,|\eta|) .
$$


This implies

$$
\left(W_{\gamma}^{-}(\varepsilon) \cap G_{\gamma}\right) \subset\left(\mathscr{A}_{\gamma}(\varepsilon) \cap G_{\gamma}\right) \subset\left(W_{\gamma}^{+}(\varepsilon) \cap G_{\gamma}\right) .
$$

From this, by (4.7), and assertions (1), (2) of Section 3 we obtain (1.12).

Proof of Theorem 1.2. (a) We fix $\gamma \leq-1$ and $\varepsilon>0$. It is clear that inequality (4.2) holds for all $(\xi, \eta) \in D_{\gamma}$.

Let $(\xi, \eta) \in \mathscr{B}_{\gamma}(\varepsilon) \cap H_{\gamma}$. In this case inequality (4.2) becomes

$$
\sigma(|\xi|)=\sigma(|\eta|) \leq \varepsilon
$$

Then

$$
\mathscr{B}_{\gamma}(\varepsilon) \cap H_{\gamma}=V_{\gamma}^{+}(\varepsilon) \cap H_{\gamma} .
$$

Using assertion (9) of Section 3, we see that

$$
\left(V_{\gamma}^{+}(\varepsilon) \cap H_{\gamma}\right) \subset\left(\mathscr{S}_{\gamma}(\varepsilon) \cap H_{\gamma}\right) \subset\left(V_{\gamma}^{-}(\varepsilon) \cap H_{\gamma}\right)
$$

Let $(\xi, \eta) \in G_{\gamma}$. Then by the inequality

$$
\Psi(\varphi) \geq(\sigma(|\xi|)|\xi|-\sigma(|\eta|)|\eta|)^{2}
$$

and by assertion (4) of Section 2, we conclude that $\Psi(\varphi)>0$ for all $\varphi \in[0, \pi]$. After simple calculations, we obtain

$$
\frac{\partial}{\partial \varphi}\left(\frac{\Phi(\varphi)}{\Psi(\varphi)}\right)=\frac{(\sigma(|\xi|)-\sigma(|\eta|))\left(|\xi|^{2} \sigma^{2}(|\xi|)-|\eta|^{2} \sigma^{2}(|\eta|)\right)|\xi||\eta| \sin \varphi}{\Psi^{2}(\varphi)} .
$$

By assertions (3) and (4) of Section 2, it follows that

$$
(\sigma(|\xi|)-\sigma(|\eta|))\left(|\xi|^{2} \sigma^{2}(|\xi|)-|\eta|^{2} \sigma^{2}(|\eta|)\right)<0 .
$$

Therefore

$$
\begin{aligned}
\min _{\varphi \in[0, \pi]}\left(\frac{\Phi(\varphi)}{\Psi(\varphi)}\right) & =\frac{\Phi(\pi)}{\Psi(\pi)} \\
& =\frac{\sigma(|\xi|)|\xi|^{2}+\sigma(|\eta|)|\eta|^{2}+(\sigma(|\xi|)+\sigma(|\eta|))|\xi||\eta|}{\sigma^{2}(|\xi|)|\xi|^{2}+\sigma^{2}(|\eta|)|\eta|^{2}+2 \sigma(|\xi|) \sigma(|\eta|)|\xi||\eta|}=\frac{1}{I_{\gamma}^{+}(|\xi|,|\eta|)} \\
\max _{\varphi \in[0, \pi]}\left(\frac{\Phi(\varphi)}{\Psi(\varphi)}\right) & =\frac{\Phi(0)}{\Psi(0)} \\
& =\frac{\sigma(|\xi|)|\xi|^{2}+\sigma(|\eta|)|\eta|^{2}-(\sigma(|\xi|)+\sigma(|\eta|))|\xi||\eta|}{\sigma^{2}(|\xi|)|\xi|^{2}+\sigma^{2}(|\eta|)|\eta|^{2}-2 \sigma(|\xi|) \sigma(|\eta|)|\xi||\eta|}=\frac{1}{I_{\gamma}^{-}(|\xi|,|\eta|)}
\end{aligned}
$$


14 Some elementary inequalities in gas dynamics equation

Thus for all $(\xi, \eta) \in G_{\gamma}$,

$$
\frac{1}{I_{\gamma}^{+}(|\xi|,|\eta|)} \leq \frac{\langle\sigma(|\xi|) \xi-\sigma(|\eta|) \eta, \xi-\eta\rangle}{|\sigma(|\xi|) \xi-\sigma(|\eta|) \eta|^{2}} \leq \frac{1}{I_{\gamma}^{-}(|\xi|,|\eta|)} .
$$

This implies that

$$
\left(V_{\gamma}^{+}(\varepsilon) \cap G_{\gamma}\right) \subset\left(\mathscr{P}_{\gamma}(\varepsilon) \cap G_{\gamma}\right) \subset\left(V_{\gamma}^{-}(\varepsilon) \cap G_{\gamma}\right) .
$$

From this, by (4.15) and assertion (6) of Section 3, we obtain (1.13) and (1.14).

(b) We fix $\gamma>-1$ and $\varepsilon>0$. It is clear that the inequality (4.2) holds for all $(\xi, \eta) \in Q_{\gamma}$. By assertion (5) of Section 2, $Q_{\gamma} \neq D_{\gamma}$.

Let $(\xi, \eta) \in \mathscr{B}_{\gamma}(\varepsilon) \cap P_{\gamma}$. Similarly we establish that $P_{\gamma} \neq H_{\gamma}$. We have

$$
\begin{aligned}
\Psi(\varphi) & =\sigma^{2}(|\xi|)|\xi|^{2}+\sigma^{2}(|\eta|)|\eta|^{2}-2 \sigma(|\xi|) \sigma(|\eta|)|\xi||\eta| \cos \varphi=2 \sigma^{2}(|\xi|)|\xi|^{2}(1-\cos \varphi) \\
\Phi(\varphi) & =\sigma(|\xi|)|\xi|^{2}+\sigma(|\eta|)|\eta|^{2}-(\sigma(|\xi|)+\sigma(|\eta|))|\xi||\eta| \cos \varphi \\
& =\sigma(|\xi|)|\xi|^{2}+\sigma(|\xi|)|\xi||\eta|-\sigma(|\xi|)|\xi||\eta| \cos \varphi-\sigma(|\xi|)|\xi|^{2} \cos \varphi \\
& =\sigma(|\xi|)|\xi|(|\xi|+|\eta|)(1-\cos \varphi) .
\end{aligned}
$$
find

It is easy to see that $\cos \varphi \neq 1$. Indeed, if $\cos \varphi=1$, then $\xi \sigma(|\xi|)=\eta \sigma(|\eta|)$. Next, we

$$
\frac{\Psi(\varphi)}{\Phi(\varphi)}=\frac{2|\xi| \sigma(|\xi|)}{|\xi|+|\eta|}=I_{\gamma}^{+}(|\xi|,|\eta|) .
$$

Thus inequality (4.2) assumes the form

$$
I_{\gamma}^{+}(|\xi|,|\eta|) \leq \varepsilon
$$

Then

$$
\mathscr{B}_{\gamma}(\varepsilon) \cap P_{\gamma}=V_{\gamma}^{+}(\varepsilon) \cap P_{\gamma} .
$$

Let $(\xi, \eta) \in U_{\gamma}^{+}$. By assertion (3) of Section 2 we find that inequality (4.18) is valid. Therefore inequalities (4.20) are true. Hence

$$
\left(V_{\gamma}^{+}(\varepsilon) \cap U_{\gamma}^{+}\right) \subset\left(\mathscr{B}_{\gamma}(\varepsilon) \cap U_{\gamma}^{+}\right) \subset\left(V_{\gamma}^{-}(\varepsilon) \cap U_{\gamma}^{+}\right) .
$$

Consider the remaining case $(\xi, \eta) \in U_{\gamma}^{-}$. Observe that the set $U_{\gamma}^{-}$is not empty. It is easy to see that

$$
(\sigma(|\xi|)-\sigma(|\eta|))\left(|\xi|^{2} \sigma^{2}(|\xi|)-|\eta|^{2} \sigma^{2}(|\eta|)\right)>0 .
$$

Hence for all $(\xi, \eta) \in U_{\gamma}^{-}$,

$$
\frac{1}{I_{\gamma}^{-}(|\xi|,|\eta|)} \leq \frac{\langle\sigma(|\xi|) \xi-\sigma(|\eta|) \eta, \xi-\eta\rangle}{|\sigma(|\xi|) \xi-\sigma(|\eta|) \eta|^{2}} \leq \frac{1}{I_{\gamma}^{+}(|\xi|,|\eta|)}
$$


which implies that

$$
\left(\mathscr{B}_{\gamma}(\varepsilon) \cap U_{\gamma}^{-}\right) \subset\left(V_{\gamma}^{+}(\varepsilon) \cap U_{\gamma}^{-}\right) .
$$

From this, by (4.25) and (4.26),

$$
F_{\gamma}^{+}(\varepsilon) \subset \mathscr{B}_{\gamma}(\varepsilon) \subset F_{\gamma}^{-}(\varepsilon) .
$$

It is not hard to establish that

$$
W_{\gamma}^{-}(0) \subset\left(P_{\gamma} \cup Q_{\gamma} \cup U_{\gamma}^{+}\right), \quad\left(P_{\gamma} \cup Q_{\gamma} \cup U_{\gamma}^{+} \cup U_{\gamma}^{-}\right)=\Omega_{\gamma} \times \Omega_{\gamma} .
$$

Then, using assertion (9) of Section 3, we find

$$
\left(V_{\gamma}^{+}(\varepsilon) \cap W_{\gamma}^{-}(0)\right) \subset F_{\gamma}^{+}(\varepsilon), \quad F_{\gamma}^{-}(\varepsilon) \subset\left(V_{\gamma}^{-}(\varepsilon) \cup Q_{\gamma}\right) .
$$

From this, by assertion (6) of Section 3 we obtain (1.15).

\section{Properties of $x_{\gamma}(\varepsilon)$}

For every $\gamma \in \mathbb{R}$ and $\varepsilon \in(0,1)$ we set

$$
\begin{gathered}
X_{\gamma}(\varepsilon)=\left\{x \in \Sigma_{\gamma}: \exists y \in \Sigma_{\gamma}, I_{\gamma}^{+}(x, y) \geq \varepsilon\right\}, \\
x_{\gamma}(\varepsilon)=\sup _{x} X_{\gamma}(\varepsilon) .
\end{gathered}
$$

If $x_{\gamma}(\varepsilon) \in \Sigma_{\gamma}$, then the following relations are true:

$$
\begin{gathered}
W_{\gamma}^{+}(\varepsilon) \subset\left\{(\xi, \eta) \in \Omega_{\gamma} \times \Omega_{\gamma}:|\xi| \leq x_{\gamma}(\varepsilon),|\eta| \leq x_{\gamma}(\varepsilon)\right\}, \\
\left\{(\xi, \eta) \in \Omega_{\gamma} \times \Omega_{\gamma}:|\xi| \geq x_{\gamma}(\varepsilon) \text { or }|\eta| \geq x_{\gamma}(\varepsilon)\right\} \subset V_{\gamma}^{+}(\varepsilon) .
\end{gathered}
$$

For every $\gamma \in \mathbb{R}$ we will study the function $x_{\gamma}(\varepsilon)$ of variable $\varepsilon$. Let $\gamma \in \mathbb{R}$ and $\varepsilon \in(0,1)$. Then

$$
I_{\gamma}^{+}\left(0, r_{\gamma}(\varepsilon)\right)=\sigma\left(r_{\gamma}(\varepsilon)\right)=\varepsilon
$$

where $r_{\gamma}(\varepsilon)$ is defined in Section 2. From this, $r_{\gamma}(\varepsilon) \in X_{\gamma}(\varepsilon)$. Therefore for every $\gamma \in \mathbb{R}$ the function $x_{\gamma}(\varepsilon)$ is defined everywhere on $(0,1)$. Moreover, for every $\gamma \in \mathbb{R}$,

$$
r_{\gamma}(\varepsilon) \leq x_{\gamma}(\varepsilon) \quad \forall \varepsilon \in(0,1)
$$

As above, let $s_{\gamma}(\varepsilon)$ be a unique positive solution of $(2.1)$ for every $\gamma \in \mathbb{R}$ and $\varepsilon \in(0,1)$. For $\gamma>1$ we put

$$
\widehat{\varepsilon}_{\gamma}=\max _{y \in[0, \sqrt{2 /(\gamma-1)}]} I_{\gamma}^{+}\left(\sqrt{\frac{2}{\gamma-1}}, y\right) .
$$


16 Some elementary inequalities in gas dynamics equation

The function $x_{\gamma}(\varepsilon)$ has the following properties.

(1) Let $\gamma>1$. Then

$$
\begin{aligned}
& x_{\gamma}(\varepsilon)=\sqrt{\frac{2}{\gamma-1}} \quad \forall \varepsilon \in\left(0, \hat{\varepsilon}_{\gamma}\right], \\
& x_{\gamma}(\varepsilon)<\sqrt{\frac{2}{\gamma-1}} \quad \forall \varepsilon \in\left(\hat{\varepsilon}_{\gamma}, 1\right) .
\end{aligned}
$$

(2) Let

$$
\gamma \in(-\infty, 1], \quad \varepsilon \in(0,1)
$$

or

$$
\gamma \in(1,+\infty), \quad \varepsilon \in\left(\widehat{\varepsilon}_{\gamma}, 1\right)
$$

Then $x_{\gamma}(\varepsilon) \in \Sigma_{\gamma}$ and

$$
I_{\gamma}^{+}\left(x_{\gamma}(\varepsilon), s_{\gamma}(\varepsilon)\right)=\varepsilon
$$

(3) For every $\gamma>1$ we have

$$
\lim _{\varepsilon \rightarrow \hat{\varepsilon}_{\gamma}+0} x_{\gamma}(\varepsilon)=\sqrt{\frac{2}{\gamma-1}} .
$$

(4) The function $x_{\gamma}(\varepsilon)$ is strictly decreasing on $(0,1)$ for $\gamma \leq 1$ and strictly decreasing on $\left(\hat{\varepsilon}_{\gamma}, 1\right)$ for $\gamma>1$. Moreover,

$$
x_{\gamma}^{\prime}(\varepsilon)=\frac{x_{\gamma}(\varepsilon)+s_{\gamma}(\varepsilon)}{\theta^{\prime}\left(x_{\gamma}(\varepsilon)\right)-\varepsilon}<0
$$

for every $\gamma$ and $\varepsilon$, satisfying (5.8) or (5.9).

(5) (a) If $\gamma \in(-\infty, 1]$, then the function $x_{\gamma}(\varepsilon) \in C^{\infty}(0,1)$.

(b) If $\gamma \in(1,2]$, then the function $x_{\gamma}(\varepsilon) \in C^{\infty}\left(\left(0, \hat{\varepsilon}_{\gamma}\right) \cup\left(\widehat{\varepsilon}_{\gamma}, 1\right)\right)$ and it is continuous at the point $\hat{\varepsilon}_{\gamma}$;

(c) If $\gamma \in(2,3]$, then the function $x_{\gamma}(\varepsilon) \in C^{\infty}\left(\left(0, \hat{\varepsilon}_{\gamma}\right) \cup\left(\hat{\varepsilon}_{\gamma}, 1\right)\right)$ and it has the continuous derivative at the point $\hat{\varepsilon}_{\gamma}$;

(d) If $\gamma \in(3,+\infty]$, then the function $x_{\gamma}(\varepsilon) \in C^{\infty}\left(\left(0, \hat{\varepsilon}_{\gamma}\right) \cup\left(\hat{\varepsilon}_{\gamma}, 1\right)\right)$ and it has the second continuous derivative at the point $\hat{\varepsilon}_{\gamma}$.

(6) For every $\gamma \in \mathbb{R}$ we have

$$
\lim _{\varepsilon \rightarrow 1-0} x_{\gamma}(\varepsilon)=0
$$

(7) For every $\gamma \leq 1$ we have

$$
\lim _{\varepsilon \rightarrow 0+} x_{\gamma}(\varepsilon)=+\infty
$$


(8) (a) If $\gamma \in(-\infty,-1)$, then

$$
\lim _{\varepsilon \rightarrow 0+} x_{\gamma}(\varepsilon) \varepsilon^{-\alpha}=0 \quad \text { for every } \alpha<\frac{\gamma-1}{2} .
$$

(b) If $\gamma=-1$, then

$$
\lim _{\varepsilon \rightarrow 0+} x_{\gamma}(\varepsilon) \varepsilon=2 .
$$

(c) If $\gamma \in(-1,1)$, then

$$
\lim _{\varepsilon \rightarrow 0+} x_{\gamma}(\varepsilon) \varepsilon=\left(\frac{\gamma+1}{2}\right)^{(\gamma+1) /(2 \gamma-2)} .
$$

(d) If $\gamma=1$, then

$$
\lim _{\varepsilon \rightarrow 0+} x_{\gamma}(\varepsilon) \varepsilon=\exp \left\{-\frac{1}{2}\right\}
$$

(9) For every $\gamma \in \mathbb{R}$ we have

$$
\lim _{\varepsilon \rightarrow 1-0} \frac{x_{\gamma}(\varepsilon)}{(1-\varepsilon)^{\alpha}}=+\infty \quad \text { for every } \alpha>\frac{1}{2}
$$

Proof of property (1). Let $\gamma>1$. We set

$$
\alpha(y)=I_{\gamma}^{+}\left(\sqrt{\frac{2}{\gamma-1}}, y\right)=\frac{\theta(y)}{y+\sqrt{2 /(\gamma-1)}} .
$$

It is easy to see that the function $\alpha(y)$ is positive on $(0, \sqrt{2 /(\gamma-1)})$ and it is continuous on $[0, \sqrt{2 /(\gamma-1)}]$. Therefore there exists

$$
\widehat{\varepsilon}_{\gamma}=\max _{y \in[0, \sqrt{2 /(\gamma-1)}]} \alpha(y)>0
$$

Next,

$$
\alpha(y) \leq \frac{y}{y+\sqrt{2 /(\gamma-1)}}<1 \quad \forall y \in\left[0, \sqrt{\frac{2}{\gamma-1}}\right]
$$

Hence $\widehat{\varepsilon}_{\gamma}<1$. Therefore for every $\varepsilon \in\left(0, \hat{\varepsilon}_{\gamma}\right]$ the equation

$$
\alpha(y)=\varepsilon
$$

has at the least one solution $y_{0} \in(0, \sqrt{2 /(\gamma-1)})$. Otherwise the equation does not have any solution. 
18 Some elementary inequalities in gas dynamics equation

Fix $\varepsilon \in\left(0, \hat{\varepsilon}_{\gamma}\right]$ and $x \in \Sigma_{\gamma}$. Let $y_{0} \in \Sigma_{\gamma}$ be a solution of (5.23). We have

$$
\varepsilon=\alpha\left(y_{0}\right)=\frac{\theta\left(y_{0}\right)}{y_{0}+\sqrt{2 /(\gamma-1)}} \leq \frac{\theta(x)+\theta\left(y_{0}\right)}{x+y_{0}}=I_{\gamma}^{+}\left(x, y_{0}\right) .
$$

From this $x \in X_{\gamma}(\varepsilon)$. Hence $X_{\gamma}(\varepsilon)=\Sigma_{\gamma}$ for all $\varepsilon \in\left(0, \hat{\varepsilon}_{\gamma}\right]$. This proves (5.6).

Now we prove (5.7). Fix $\varepsilon \in\left(\widehat{\varepsilon}_{\gamma}, 1\right)$. Suppose that

$$
x_{\gamma}(\varepsilon)=\sqrt{\frac{2}{\gamma-1}} .
$$

Then for arbitrary $n \in \mathbb{N}$ there exists a number $x_{n} \in X_{\gamma}(\varepsilon)$ such that

$$
\sqrt{\frac{2}{\gamma-1}}-\frac{1}{n}<x_{n}
$$

Moreover,

$$
\lim _{n \rightarrow \infty} x_{n}=\sqrt{\frac{2}{\gamma-1}}
$$

and for arbitrary $n \in \mathbb{N}$ there exists $y_{n} \in \Sigma_{\gamma}$ satisfying the inequality

$$
I_{\gamma}^{+}\left(x_{n}, y_{n}\right) \geq \varepsilon
$$

which implies

$$
\theta\left(x_{n}\right)-\varepsilon x_{n} \geq \varepsilon y_{n}-\theta\left(y_{n}\right) .
$$

Further, we have

$$
\alpha\left(y_{n}\right)=\frac{\theta\left(y_{n}\right)}{y_{n}+\sqrt{2 /(\gamma-1)}} \leq \widehat{\varepsilon_{\gamma}} \quad \forall n \in \mathbb{N} .
$$

Then

$$
\theta\left(y_{n}\right) \leq \hat{\varepsilon}_{\gamma}\left(y_{n}+\sqrt{\frac{2}{\gamma-1}}\right) \quad \forall n \in \mathbb{N} .
$$

Using (5.29), for all $n \in \mathbb{N}$ we deduce

$$
\begin{aligned}
\theta\left(x_{n}\right)-\varepsilon x_{n} & \geq \varepsilon y_{n}-\theta\left(y_{n}\right) \\
& \geq \varepsilon y_{n}-\hat{\varepsilon}_{\gamma}\left(y_{n}+\sqrt{\frac{2}{\gamma-1}}\right) \geq-\hat{\varepsilon}_{\gamma} \sqrt{\frac{2}{\gamma-1}} .
\end{aligned}
$$


Letting $n \rightarrow \infty$ in the inequality

$$
\theta\left(x_{n}\right)-\varepsilon x_{n} \geq-\widehat{\varepsilon}_{\gamma} \sqrt{\frac{2}{\gamma-1}},
$$

we see that $\varepsilon \leq \hat{\varepsilon}_{\gamma}$ and we arrive at a contradiction.

Further we will need the following lemma.

LEMma 5.1. If (5.8) or (5.9) holds, then $x_{\gamma}(\varepsilon) \in \Sigma_{\gamma}$ and there exists a number $y_{\gamma}(\varepsilon) \in \Sigma_{\gamma}$ such that

$$
I_{\gamma}^{+}\left(x_{\gamma}(\varepsilon), y_{\gamma}(\varepsilon)\right)=\varepsilon
$$

Proof. Show that the set $X_{\gamma}(\varepsilon)$ is compact for every $\gamma$ and every $\epsilon$ satisfying (5.8) or (5.9). Introduce the set

$$
Z_{\gamma}(\varepsilon)=\left\{(x, y) \in \Sigma_{\gamma} \times \Sigma_{\gamma}: I_{\gamma}^{+}(x, y) \geq \varepsilon\right\}
$$

Let $\pi: \mathbb{R}^{2} \rightarrow \mathbb{R}, \pi(x, y)=x$ be natural projection. It is clear that $\pi\left(Z_{\gamma}(\varepsilon)\right)=X_{\gamma}(\varepsilon)$.

Assume that (5.8) holds. The set $Z_{\gamma}(\varepsilon)$ is closed since the function $I_{\gamma}^{+}(x, y)$ is continuous. The set $Z_{\gamma}(\varepsilon)$ is bounded. Indeed, we can find a sequence $Z_{\gamma}(\varepsilon) \ni\left(x_{n}, y_{n}\right) \rightarrow \infty$. Assume that $x_{n} \rightarrow \infty$. Then for the bounded subsequence of $\left\{y_{n}\right\}$ we have

$$
\varepsilon \leq I_{\gamma}^{+}\left(x_{n}, y_{n}\right)=\frac{x_{n} \sigma\left(x_{n}\right)+y_{n} \sigma\left(y_{n}\right)}{x_{n}+y_{n}} \leq \frac{x_{n} \sigma\left(x_{n}\right)+y_{n}}{x_{n}}
$$

The right part of this inequality tends to zero as $n \rightarrow \infty$. Thus we obtain a contradiction to (5.8). For an unbounded subsequence of $\left\{y_{n}\right\}$ we have

$$
\varepsilon \leq I_{\gamma}^{+}\left(x_{n}, y_{n}\right) \leq \sigma\left(x_{n}\right)+\sigma\left(y_{n}\right) \text {. }
$$

The right part of this inequality tends to zero as $n \rightarrow \infty$. Again we obtain a contradiction to (5.8). Hence $Z_{\gamma}(\varepsilon)$ is bounded. Therefore $Z_{\gamma}(\varepsilon)$ is compact. Because the mapping $\pi$ is continuous, the set $X_{\gamma}(\varepsilon)=\pi\left(Z_{\gamma}(\varepsilon)\right)$ is compact too.

Assume that (5.9) holds. By (5.7) it follows that $\overline{Z_{\gamma}(\varepsilon)} \subset \Sigma_{\gamma} \times \Sigma_{\gamma}$. Here $\overline{Z_{\gamma}(\varepsilon)}$ denotes the closure of $Z_{\gamma}(\varepsilon)$. Since the function $I_{\gamma}^{+}(x, y)$ is continuous, $Z_{\gamma}(\varepsilon)$ is compact. Therefore $X_{\gamma}(\varepsilon)$ is compact too.

Similarly we establish that the set

$$
\bar{X}_{\gamma}(\varepsilon)=\left\{x \in \Sigma_{\gamma}: \exists y \in \Sigma_{\gamma}, I_{\gamma}^{+}(x, y)=\varepsilon\right\}
$$

is compact for every $\gamma$ and $\epsilon$ satisfying (5.8) or (5.9).

We fix $\gamma$ and $\epsilon$ satisfying (5.8) or (5.9). Prove that

$$
\max _{x} X_{\gamma}(\varepsilon)=\max _{x} \bar{X}_{\gamma}(\varepsilon) .
$$


20 Some elementary inequalities in gas dynamics equation

We set

$$
a=\max _{x} X_{\gamma}(\varepsilon), \quad b=\max _{x} \bar{X}_{\gamma}(\varepsilon) .
$$

Obviously, $a \geq b$. Show that $a \leq b$. Since $a \in X_{\gamma}(\varepsilon)$, there exists a number $y_{0} \in \Sigma_{\gamma}$ such that

$$
I_{\gamma}^{+}\left(a, y_{0}\right) \geq \varepsilon
$$

Assume that

$$
I_{\gamma}^{+}\left(a, y_{0}\right)=\varepsilon
$$

Then $a \in \bar{X}_{\gamma}(\varepsilon)$ and hence $a \leq b$.

Now we assume

$$
I_{\gamma}^{+}\left(a, y_{0}\right)>\varepsilon
$$

For $\gamma \leq 1$ we have

$$
\lim _{x \rightarrow+\infty} I_{\gamma}^{+}\left(x, y_{0}\right)=0
$$

Since the function $I_{\gamma}^{+}(x, y)$ is continuous, there exists a number $x^{\prime}>a$ such that

$$
I_{\gamma}^{+}\left(x^{\prime}, y_{0}\right)=\varepsilon
$$

Then $x^{\prime} \in \bar{X}_{\gamma}(\varepsilon)$. Hence $a<x^{\prime} \leq b$ and we arrive at a contradiction. For $\gamma>1$ we have

$$
I_{\gamma}^{+}\left(\sqrt{\frac{2}{\gamma-1}}, y_{0}\right) \leq \hat{\varepsilon}_{\gamma}<\varepsilon .
$$

Then there exists a number $x^{\prime} \in(a, \sqrt{2 /(\gamma-1)})$ satisfying (5.45). Hence $x^{\prime} \in \bar{X}_{\gamma}(\varepsilon)$. Therefore $a<x^{\prime} \leq b$ and we arrive at a contradiction.

Thus we establish that

$$
x_{\gamma}(\varepsilon)=\max _{x} \bar{X}_{\gamma}(\varepsilon)
$$

and arrive at the desired result.

Proof of properties (2)-(5). Fix $\gamma$ and $\varepsilon_{0}$ satisfying (5.8) or (5.9). By Lemma 5.1 the number $x_{\gamma}\left(\varepsilon_{0}\right) \in \Sigma_{\gamma}$ and there exists a number $y_{\gamma}\left(\varepsilon_{0}\right) \in \Sigma_{\gamma}$ such that

$$
I_{\gamma}^{+}\left(x_{\gamma}\left(\varepsilon_{0}\right), y_{\gamma}\left(\varepsilon_{0}\right)\right)=\varepsilon_{0} .
$$

We set

$$
F(x, y, \varepsilon)=I_{\gamma}^{+}(x, y)-\varepsilon \text {. }
$$


Observe that the function $F(x, y, \varepsilon)$ is $C^{\infty}$-differentiable in some neighborhood $U \subset \mathbb{R}^{3}$ of the point $p_{0}=\left(x_{\gamma}\left(\varepsilon_{0}\right), y_{\gamma}\left(\varepsilon_{0}\right), \varepsilon_{0}\right)$ and $F\left(p_{0}\right)=0$. We have

$$
\frac{\partial F}{\partial x}\left(p_{0}\right)=\frac{\theta^{\prime}\left(x_{\gamma}\left(\varepsilon_{0}\right)\right)-I_{\gamma}^{+}\left(x_{\gamma}\left(\varepsilon_{0}\right), y_{\gamma}\left(\varepsilon_{0}\right)\right)}{x_{\gamma}\left(\varepsilon_{0}\right)+y_{\gamma}\left(\varepsilon_{0}\right)}=\frac{\theta^{\prime}\left(x_{\gamma}\left(\varepsilon_{0}\right)\right)-\varepsilon_{0}}{x_{\gamma}\left(\varepsilon_{0}\right)+y_{\gamma}\left(\varepsilon_{0}\right)} .
$$

By assertion (8) of Section 2, $0<s_{\gamma}\left(\varepsilon_{0}\right)<r_{\gamma}\left(\varepsilon_{0}\right)$. Therefore the inequality $r_{\gamma}\left(\varepsilon_{0}\right) \leq x_{\gamma}\left(\varepsilon_{0}\right)$ yields

$$
\frac{\partial F}{\partial x}\left(p_{0}\right)<0
$$

By the well-known implicit function theorem, there exist a 3-dimensional interval $I=$ $I_{x} \times I_{y} \times I_{\varepsilon} \subset U$ and a function $f \in C^{\infty}\left(I_{y} \times I_{\varepsilon}\right)$ such that for all $(x, y, \varepsilon) \in I_{x} \times I_{y} \times I_{\varepsilon}$,

$$
F(x, y, \varepsilon)=0 \Longleftrightarrow x=f(y, \varepsilon) .
$$

Here

$$
\begin{gathered}
I_{x}=\left\{x \in \mathbb{R}:\left|x-x_{\gamma}\left(\varepsilon_{0}\right)\right|<a\right\}, \quad I_{y}=\left\{y \in \mathbb{R}:\left|y-y_{\gamma}\left(\varepsilon_{0}\right)\right|<b\right\}, \\
I_{\varepsilon}=\left\{\varepsilon \in \mathbb{R}:\left|\varepsilon-\varepsilon_{0}\right|<c\right\} .
\end{gathered}
$$

Moreover,

$$
\begin{aligned}
& \frac{\partial f}{\partial y}\left(y_{\gamma}\left(\varepsilon_{0}\right), \varepsilon_{0}\right)=-\left[F_{x}^{\prime}\left(p_{0}\right)\right]^{-1}\left[F_{y}^{\prime}\left(p_{0}\right)\right]=-\frac{\theta^{\prime}\left(y_{\gamma}\left(\varepsilon_{0}\right)\right)-\varepsilon_{0}}{\theta^{\prime}\left(x_{\gamma}\left(\varepsilon_{0}\right)\right)-\varepsilon_{0}}, \\
& \frac{\partial f}{\partial \varepsilon}\left(y_{\gamma}\left(\varepsilon_{0}\right), \varepsilon_{0}\right)=-\left[F_{x}^{\prime}\left(p_{0}\right)\right]^{-1}\left[F_{\varepsilon}^{\prime}\left(p_{0}\right)\right]=\frac{x_{\gamma}\left(\varepsilon_{0}\right)+y_{\gamma}\left(\varepsilon_{0}\right)}{\theta^{\prime}\left(x_{\gamma}\left(\varepsilon_{0}\right)\right)-\varepsilon_{0}} .
\end{aligned}
$$

It is easy to see that at the point $y_{\gamma}\left(\varepsilon_{0}\right)$ the function $x=f\left(y, \varepsilon_{0}\right)$ reaches a maximum on $I_{y}$. Therefore

$$
\frac{\partial f}{\partial y}\left(y_{\gamma}\left(\varepsilon_{0}\right), \varepsilon_{0}\right)=0
$$

From this

$$
\theta^{\prime}\left(y_{\gamma}\left(\varepsilon_{0}\right)\right)=\varepsilon_{0}
$$

Hence $y_{\gamma}\left(\varepsilon_{0}\right)=s_{\gamma}\left(\varepsilon_{0}\right)$ and property (2) is proved.

Further, we set

$$
G(y, \varepsilon)=\theta^{\prime}(y)-\varepsilon
$$

Observe that the function $G(y, \varepsilon)$ is $C^{\infty}$-differentiable in some neighborhood $V \subset \mathbb{R}^{2}$ of the point $q_{0}=\left(s_{\gamma}\left(\varepsilon_{0}\right), \varepsilon_{0}\right)$ and $G\left(q_{0}\right)=0$. By assertions $(6)-(8)$ of Section 2 we have

$$
\frac{\partial G}{\partial y}\left(q_{0}\right)=\theta^{\prime \prime}\left(s_{\gamma}\left(\varepsilon_{0}\right)\right)<0 .
$$


22 Some elementary inequalities in gas dynamics equation

By the implicit function theorem, the function $s_{\gamma}(\varepsilon)$ is $C^{\infty}$-differentiable at the point $\varepsilon_{0}$. Therefore there is an interval

$$
I_{\varepsilon}^{\prime}=\left\{\varepsilon \in \mathbb{R}:\left|\varepsilon-\varepsilon_{0}\right|\left\langle c^{\prime}\right\} \subset I_{\varepsilon}\right.
$$

such that

$$
s_{\gamma}(\varepsilon) \in I_{y} \quad \forall \varepsilon \in I_{\varepsilon}^{\prime} .
$$

Hence for all $(x, \varepsilon) \in I_{x} \times I_{\varepsilon}^{\prime}$,

$$
F\left(x, s_{\gamma}(\varepsilon), \varepsilon\right)=0 \Longleftrightarrow x=f\left(s_{\gamma}(\varepsilon), \varepsilon\right) .
$$

We fix $\varepsilon \in I_{\varepsilon}^{\prime}$. Next,

$$
x=f\left(s_{\gamma}(\varepsilon), \varepsilon\right)
$$

and hence

$$
F\left(x, s_{\gamma}(\varepsilon), \varepsilon\right)=0
$$

Rewrite the latter equality in the form

$$
\mu(x)=-\mu\left(s_{\gamma}(\varepsilon)\right)
$$

where

$$
\mu(t)=\mu(t, \varepsilon)=\theta(t)-t \varepsilon
$$

We have

$$
\mu^{\prime}(t)=\theta^{\prime}(t)-\varepsilon
$$

By assertion (8) of Section 2 we conclude that the function $\mu(t)$ is strictly increasing on $\left(0, s_{\gamma}(\varepsilon)\right)$ and strictly decreasing on $\left(s_{\gamma}(\varepsilon),+\infty\right) \cap \Sigma_{\gamma}$. Moreover, $\mu(0)=\mu\left(r_{\gamma}(\varepsilon)\right)=0$ and by property (2), $\mu\left(x_{\gamma}(\varepsilon)\right)=-\mu\left(s_{\gamma}(\varepsilon)\right)$. Then it is not hard to check that $x=x_{\gamma}(\varepsilon)$. Thus

$$
x_{\gamma}(\varepsilon)=f\left(s_{\gamma}(\varepsilon), \varepsilon\right) \quad \forall \varepsilon \in I_{\varepsilon}^{\prime} .
$$

Hence the function $x_{\gamma}(\varepsilon)$ is $C^{\infty}$-differentiable at the point $\varepsilon_{0}$ and

$$
x_{\gamma}^{\prime}\left(\varepsilon_{0}\right)=\frac{\partial f}{\partial y}\left(s_{\gamma}\left(\varepsilon_{0}\right), \varepsilon_{0}\right) s_{\gamma}^{\prime}\left(\varepsilon_{0}\right)+\frac{\partial f}{\partial \varepsilon}\left(s_{\gamma}\left(\varepsilon_{0}\right), \varepsilon_{0}\right)=\frac{\partial f}{\partial \varepsilon}\left(s_{\gamma}\left(\varepsilon_{0}\right), \varepsilon_{0}\right)=\frac{x_{\gamma}\left(\varepsilon_{0}\right)+s_{\gamma}\left(\varepsilon_{0}\right)}{\theta^{\prime}\left(x_{\gamma}\left(\varepsilon_{0}\right)\right)-\varepsilon_{0}}<0 .
$$

This proves property (4).

Let $\gamma>1$. We show that

$$
\lim _{\varepsilon \rightarrow \widehat{\varepsilon}_{y}+0} x_{\gamma}(\varepsilon)=\sqrt{\frac{2}{\gamma-1}}
$$


Let $y_{0} \in \Sigma_{\gamma}$ be a solution of the equation

$$
\alpha(y)=\hat{\varepsilon}_{\gamma}
$$

Here, as above,

$$
\alpha(y)=I_{\gamma}^{+}\left(\sqrt{\frac{2}{\gamma-1}}, y\right) .
$$

Then

$$
\begin{gathered}
\frac{\theta\left(y_{0}\right)}{y_{0}+\sqrt{2 /(\gamma-1)}}=\widehat{\varepsilon}_{\gamma}, \\
\alpha^{\prime}\left(y_{0}\right)=\frac{\theta^{\prime}\left(y_{0}\right)\left(y_{0}+\sqrt{2 /(\gamma-1)}\right)-\theta\left(y_{0}\right)}{\left(y_{0}+\sqrt{2 /(\gamma-1)}\right)^{2}}=0 .
\end{gathered}
$$

From this

$$
\theta\left(y_{0}\right)=\theta^{\prime}\left(y_{0}\right)\left(y_{0}+\sqrt{\frac{2}{\gamma-1}}\right) .
$$

Using (5.72), we conclude that

$$
\theta^{\prime}\left(y_{0}\right)=\widehat{\varepsilon}_{\gamma}
$$

that is, $y_{0}=s_{\gamma}\left(\hat{\varepsilon}_{\gamma}\right)$.

We rewrite the equality

$$
I_{\gamma}^{+}\left(x_{\gamma}(\varepsilon), s_{\gamma}(\varepsilon)\right)=\varepsilon
$$

in the form

$$
\theta\left(x_{\gamma}(\varepsilon)\right)-x_{\gamma}(\varepsilon) \varepsilon=-\left(\theta\left(s_{\gamma}(\varepsilon)\right)-s_{\gamma}(\varepsilon) \varepsilon\right) .
$$

Using (5.72), we obtain

$$
\lim _{\varepsilon \rightarrow \hat{\varepsilon}_{\gamma}+0}\left(\theta\left(x_{\gamma}(\varepsilon)\right)-x_{\gamma}(\varepsilon) \varepsilon\right)=-\left(\theta\left(s_{\gamma}\left(\widehat{\varepsilon}_{\gamma}\right)\right)-s_{\gamma}\left(\widehat{\varepsilon}_{\gamma}\right) \hat{\varepsilon}_{\gamma}\right)=-\widehat{\varepsilon}_{\gamma} \sqrt{\frac{2}{\gamma-1}} .
$$


24 Some elementary inequalities in gas dynamics equation

Thus

$$
\lim _{\varepsilon \rightarrow \hat{\varepsilon}+0} \mu\left(x_{\gamma}(\varepsilon), \varepsilon\right)=-\hat{\varepsilon}_{\gamma} \sqrt{\frac{2}{\gamma-1}} .
$$

Suppose that (5.69) is not true. That is, for some sequence $\varepsilon_{i} \rightarrow \widehat{\varepsilon}_{\gamma}+0$ of numbers the inequality

$$
x_{\gamma}\left(\varepsilon_{i}\right) \leq \sqrt{\frac{2}{\gamma-1}}-m
$$

holds with some constant $m>0$. Note that $x_{\gamma}(\varepsilon) \in\left[r_{\gamma}(\varepsilon), \sqrt{(2 / \gamma-1)}\right)$ for every $\varepsilon \in\left(\hat{\varepsilon}_{\gamma}, 1\right)$. By assertion (8) of Section 2 it follows that the function $\mu(t)$ is strictly decreasing on $\left[r_{\gamma}(\varepsilon), \sqrt{(2 / \gamma-1)}\right)$. We have

$$
\mu\left(x_{\gamma}\left(\varepsilon_{i}\right), \varepsilon_{i}\right) \geq \mu\left(\sqrt{\frac{2}{\gamma-1}}-m, \varepsilon_{i}\right)>-\left(\sqrt{\frac{2}{\gamma-1}}-m\right) \varepsilon_{i} .
$$

Letting $\varepsilon_{i} \rightarrow \widehat{\varepsilon}_{\gamma}+0$, we obtain a contradiction to (5.78). Thus property (3) is proved. Hence the function $x_{\gamma}(\varepsilon)$ is continuous at the point $\hat{\varepsilon}_{\gamma}$ for every $\gamma>1$.

For $\gamma>1$ we have

$$
A_{\gamma}=\lim _{\varepsilon \rightarrow \hat{\varepsilon}_{\gamma}+0} \frac{x_{\gamma}(\varepsilon)-x_{\gamma}\left(\widehat{\varepsilon}_{\gamma}\right)}{\varepsilon-\hat{\varepsilon}_{\gamma}}=\lim _{\varepsilon \rightarrow \hat{\varepsilon}_{\gamma}+0} x_{\gamma}^{\prime}(\varepsilon)=\lim _{\varepsilon \rightarrow \hat{\varepsilon}_{\gamma}+0} \frac{x_{\gamma}(\varepsilon)+s_{\gamma}(\varepsilon)}{\theta^{\prime}\left(x_{\gamma}(\varepsilon)\right)-\varepsilon} .
$$

By assertion (5) of Section 2 and by property (3) we obtain

$$
\begin{gathered}
A_{\gamma}=-\frac{\sqrt{2 /(\gamma-1)}+s_{\gamma}\left(\hat{\varepsilon}_{\gamma}\right)}{\hat{\varepsilon}_{\gamma}}<0 \quad \text { for } \gamma \in(1,2), \\
A_{\gamma}=-\frac{\sqrt{2 /(\gamma-1)}+s_{\gamma}\left(\hat{\varepsilon}_{\gamma}\right)}{2+\hat{\varepsilon}_{\gamma}}<0 \quad \text { for } \gamma=2, \\
A_{\gamma}=0 \quad \text { for } \gamma \in(2,+\infty) .
\end{gathered}
$$

Hence $x_{\gamma}(\varepsilon)$ is not differentiable at the point $\hat{\varepsilon}_{\gamma}$ for $\gamma \in(1,2]$ and it has the continuous derivative at the point $\widehat{\varepsilon}_{\gamma}$ for $\gamma \in(2,+\infty)$.

For $\gamma>2$ we have

$$
B_{\gamma}=\lim _{\varepsilon \rightarrow \hat{\varepsilon}_{\gamma}+0} \frac{x_{\gamma}^{\prime}(\varepsilon)-x_{\gamma}^{\prime}\left(\widehat{\varepsilon}_{\gamma}\right)}{\varepsilon-\widehat{\varepsilon}_{\gamma}}=\lim _{\varepsilon \rightarrow \hat{\varepsilon}_{\gamma}+0} \frac{x_{\gamma}(\varepsilon)+s_{\gamma}(\varepsilon)}{\left(\theta^{\prime}\left(x_{\gamma}(\varepsilon)\right)-\varepsilon\right)\left(\varepsilon-\hat{\varepsilon}_{\gamma}\right)} .
$$


Using L'Hospital rule and property (3), we find

$$
\begin{aligned}
\lim _{\varepsilon \rightarrow \hat{\varepsilon}_{\gamma}+0} & \left(1-\frac{\gamma-1}{2} x_{\gamma}^{2}(\varepsilon)\right)^{(2-\gamma) /(\gamma-1)}\left(\varepsilon-\hat{\varepsilon}_{\gamma}\right) \\
& =\lim _{\varepsilon \rightarrow \hat{\varepsilon}_{\gamma}+0} \frac{\varepsilon-\widehat{\varepsilon}_{\gamma}}{\left(1-((\gamma-1) / 2) x_{\gamma}^{2}(\varepsilon)\right)^{(\gamma-2) /(\gamma-1)}} \\
& =\lim _{\varepsilon \rightarrow \hat{\varepsilon}_{\gamma}+0} \frac{1}{-(\gamma-2) x_{\gamma}(\varepsilon) x_{\gamma}^{\prime}(\varepsilon)\left(1-((\gamma-1) / 2) x_{\gamma}^{2}(\varepsilon)\right)^{-1 /(\gamma-1)}} \\
& =-\frac{1}{\gamma-2} \lim _{\varepsilon \rightarrow \widehat{\varepsilon}_{\gamma}+0} \frac{\sigma\left(x_{\gamma}(\varepsilon)\right)\left(\theta^{\prime}\left(x_{\gamma}(\varepsilon)\right)-\varepsilon\right)}{x_{\gamma}(\varepsilon)\left(x_{\gamma}(\varepsilon)+s_{\gamma}(\varepsilon)\right)} \\
& =-\frac{1}{\gamma-2} \lim _{\varepsilon \rightarrow \hat{\varepsilon}_{\gamma}+0} \frac{\sigma\left(x_{\gamma}(\varepsilon)\right) \theta^{\prime}\left(x_{\gamma}(\varepsilon)\right)}{x_{\gamma}(\varepsilon)\left(x_{\gamma}(\varepsilon)+s_{\gamma}(\varepsilon)\right)} \\
= & -\frac{1}{\gamma-2} \lim _{\varepsilon \rightarrow \hat{\varepsilon}_{\gamma}+0} \frac{\left(1-((\gamma+1) / 2) x_{\gamma}^{2}(\varepsilon)\right)\left(1-((\gamma-1) / 2) x_{\gamma}^{2}(\varepsilon)\right)^{(3-\gamma) /(\gamma-1)}}{x_{\gamma}(\varepsilon)\left(x_{\gamma}(\varepsilon)+s_{\gamma}(\varepsilon)\right)} .
\end{aligned}
$$

Then

$$
\begin{aligned}
B_{\gamma} & =\lim _{\varepsilon \rightarrow \widehat{\varepsilon}_{\gamma}+0} \frac{x_{\gamma}(\varepsilon)+s_{\gamma}(\varepsilon)}{\theta^{\prime}\left(x_{\gamma}(\varepsilon)\right)\left(\varepsilon-\hat{\varepsilon}_{\gamma}\right)} \\
& =-(\gamma-2) \lim _{\varepsilon \rightarrow \hat{\varepsilon}_{\gamma}+0} \frac{x_{\gamma}(\varepsilon)\left(x_{\gamma}(\varepsilon)+s_{\gamma}(\varepsilon)\right)^{2}}{\left(1-((\gamma+1) / 2) x_{\gamma}^{2}(\varepsilon)\right)^{2}\left(1-((\gamma-1) / 2) x_{\gamma}^{2}(\varepsilon)\right)^{(3-\gamma) /(\gamma-1)}} .
\end{aligned}
$$

By property (3) we find

$$
\begin{aligned}
& B_{\gamma}=-\infty \quad \text { for } \gamma \in(2,3), \\
& B_{\gamma}=-\left(1+s_{\gamma}\left(\hat{\varepsilon}_{\gamma}\right)\right)^{2}<0 \quad \text { for } \gamma=3, \\
& B_{\gamma}=0 \text { for } \gamma \in(3,+\infty)
\end{aligned}
$$

Therefore the function $x_{\gamma}(\varepsilon)$ is not doubly differentiable at the point $\hat{\varepsilon}_{\gamma}$ for $\gamma \in(2,3]$ and it has second continuous derivative at the point $\hat{\varepsilon}_{\gamma}$ for $\gamma \in(3,+\infty)$. Thus property (5) is proved.

Proof of property (6). By assertion (8) of Section 2,

$$
0<s_{\gamma}(\varepsilon)<r_{\gamma}(\varepsilon)
$$

for every $\varepsilon$ and $\gamma$ satisfying (5.8) or (5.9). Letting $\varepsilon \rightarrow 1-0$ we obtain

$$
\lim _{\varepsilon \rightarrow 1-0} s_{\gamma}(\varepsilon)=0 .
$$


26 Some elementary inequalities in gas dynamics equation

Show that

$$
\lim _{\varepsilon \rightarrow 1-0} x_{\gamma}(\varepsilon)=0
$$

Indeed, suppose that this is not true, that is, there will be a number $\varepsilon_{0} \in(0,1)$ and a sequence $\varepsilon_{i} \rightarrow 1\left(\varepsilon_{0}<\varepsilon_{i}<1\right)$ such that the inequalities

$$
m \leq x_{\gamma}\left(\varepsilon_{i}\right) \leq x_{\gamma}\left(\varepsilon_{0}\right)
$$

hold with some constant $m>0$. We can consider that

$$
\lim _{\varepsilon_{i} \rightarrow 1} x_{\gamma}\left(\varepsilon_{i}\right)=a \in\left[m, x_{\gamma}\left(\varepsilon_{0}\right)\right] .
$$

Using property (2), we have

$$
1=\lim _{\varepsilon_{i} \rightarrow 1} \varepsilon_{i}=\lim _{\varepsilon_{i} \rightarrow 1} I_{\gamma}^{+}\left(x_{\gamma}\left(\varepsilon_{i}\right), s_{\gamma}\left(\varepsilon_{i}\right)\right)=I_{\gamma}^{+}(a, 0)=\sigma(a) .
$$

Then $a=0<m$ and we arrive at a contradiction.

Proof of property (7). Letting $\varepsilon \rightarrow 0+$ in the inequality $x_{\gamma}(\varepsilon) \geq r_{\gamma}(\varepsilon)$, we obtain (5.7).

Proof of property (8). (a) Let $\gamma<-1$. By assertion (8) of Section 2,

$$
0<s_{\gamma}(\varepsilon)<r_{\gamma}(\varepsilon) \quad \forall \varepsilon \in(0,1) .
$$

From this

$$
\lim _{\varepsilon \rightarrow 0+} s_{\gamma}(\varepsilon) \varepsilon^{-\alpha}=0 \quad \text { for every } \alpha<\frac{\gamma-1}{2} .
$$

We set

$$
\vartheta(t)=\left(1-\frac{\gamma+1}{2} t^{2}\right)\left(1-\frac{\gamma-1}{2} t^{2}\right)^{-1}
$$

Obviously

$$
\lim _{t \rightarrow+\infty} \vartheta(t)=\frac{\gamma+1}{\gamma-1}
$$

It is easy to see that the function $\vartheta(t)$ is strictly decreasing on $[0,+\infty)$. Therefore

$$
\vartheta(t)>\frac{\gamma+1}{\gamma-1} \quad \forall t \geq 0 .
$$

Next, for all $\varepsilon \in(0,1)$,

$$
\varepsilon=\theta^{\prime}\left(s_{\gamma}(\varepsilon)\right)=\left(1-\frac{\gamma-1}{2} s_{\gamma}^{2}(\varepsilon)\right)^{(1 /(\gamma-1))-1}\left(1-\frac{\gamma+1}{2} s_{\gamma}^{2}(\varepsilon)\right)>\sigma\left(s_{\gamma}(\varepsilon)\right) \frac{\gamma+1}{\gamma-1} .
$$


From this

$$
1<\frac{\sigma\left(s_{\gamma}(\varepsilon)\right)}{\varepsilon}<\frac{\gamma-1}{\gamma+1}
$$

for all $\varepsilon \in(0,1)$. We note that the equality $I_{\gamma}^{+}(x, y)=\varepsilon$ can be written as

$$
x\left(\frac{\sigma(x)}{\varepsilon}-1\right)=y\left(1-\frac{\sigma(y)}{\varepsilon}\right) .
$$

By (5.94) and (5.99) we obtain

$$
0=\lim _{\varepsilon \rightarrow 0+} s_{\gamma}(\varepsilon) \varepsilon^{-\alpha}\left(\frac{\sigma\left(s_{\gamma}(\varepsilon)\right)}{\varepsilon}-1\right)=\lim _{\varepsilon \rightarrow 0+} x_{\gamma}(\varepsilon) \varepsilon^{-\alpha}\left(1-\frac{\sigma\left(x_{\gamma}(\varepsilon)\right)}{\varepsilon}\right)
$$

for each $\alpha<(\gamma-1) / 2$.

Assume that there exits $\alpha<(\gamma-1) / 2$ such that

$$
\lim _{\varepsilon \rightarrow 0+} x_{\gamma}(\varepsilon) \varepsilon^{-\alpha} \neq 0 .
$$

Then for some sequence $\varepsilon_{i} \rightarrow 0$ of positive numbers the inequality

$$
x_{\gamma}\left(\varepsilon_{i}\right) \varepsilon_{i}^{-\alpha} \geq m
$$

holds with some constant $m>0$. By (5.101) we find

$$
\lim _{\varepsilon_{i} \rightarrow 0+} \frac{\sigma\left(x_{\gamma}\left(\varepsilon_{i}\right)\right)}{\varepsilon_{i}}=1 .
$$

By (5.103),

$$
\lim _{\varepsilon_{i} \rightarrow 0+} \frac{\sigma\left(x_{\gamma}\left(\varepsilon_{i}\right)\right)}{\varepsilon_{i}} \leq \lim _{\varepsilon_{i} \rightarrow 0+} \frac{\sigma\left(m \varepsilon_{i}^{\alpha}\right)}{\varepsilon_{i}}=0
$$

and we arrive at a contradiction.

(b) Let $\gamma=-1$. We have

$$
\varepsilon=\theta^{\prime}\left(s_{\gamma}(\varepsilon)\right)=\left(1+s_{\gamma}^{2}(\varepsilon)\right)^{-3 / 2} .
$$

Then

$$
s_{\gamma}(\varepsilon)=\sqrt{\varepsilon^{-2 / 3}-1}, \quad \sigma\left(s_{\gamma}(\varepsilon)\right)=\varepsilon^{1 / 3} .
$$

By property (7),

$$
\lim _{\varepsilon \rightarrow 0+} \theta\left(x_{\gamma}(\varepsilon)\right)=1 \text {. }
$$

We have

$$
1=\lim _{\varepsilon \rightarrow 0+}\left(\theta\left(s_{\gamma}(\varepsilon)\right)-s_{\gamma}(\varepsilon) \varepsilon\right)=\lim _{\varepsilon \rightarrow 0+}\left(x_{\gamma}(\varepsilon) \varepsilon-\theta\left(x_{\gamma}(\varepsilon)\right)\right) .
$$


28 Some elementary inequalities in gas dynamics equation

From this

$$
\lim _{\epsilon \rightarrow 0+} x_{\gamma}(\varepsilon) \varepsilon=2 .
$$

(c) Let $\gamma \in(-1,1)$. By property (7),

$$
\lim _{\varepsilon \rightarrow 0+} \theta\left(x_{\gamma}(\varepsilon)\right)=0 .
$$

Assertions (7) and (8) of Section 2 yield

$$
\lim _{\varepsilon \rightarrow 0+} s_{\gamma}(\varepsilon)=\sqrt{\frac{2}{\gamma+1}} .
$$

Then, using (5.111), we obtain

$$
\begin{aligned}
\left(\frac{\gamma+1}{2}\right)^{(\gamma+1) /(2 \gamma-2)} & =\theta\left(\sqrt{\frac{2}{\gamma+1}}\right)=\lim _{\varepsilon \rightarrow 0+}\left(\theta\left(s_{\gamma}(\varepsilon)\right)-s_{\gamma}(\varepsilon) \varepsilon\right) \\
& =\lim _{\varepsilon \rightarrow 0+}\left(x_{\gamma}(\varepsilon) \varepsilon-\theta\left(x_{\gamma}(\varepsilon)\right)\right)=\lim _{\epsilon \rightarrow 0+} x_{\gamma}(\varepsilon) \varepsilon .
\end{aligned}
$$

(d) The proof is analogous.

Proof of property (9). Assume that $\gamma>1$. Then

$$
x_{\gamma}(\varepsilon) \geq r_{\gamma}(\varepsilon)=\sqrt{\frac{2\left(1-\varepsilon^{\gamma-1}\right)}{\gamma-1}} .
$$

Using L'Hospital rule, we find

$$
\lim _{\varepsilon \rightarrow 1-0} \frac{1-\varepsilon^{\gamma-1}}{(1-\varepsilon)^{2 \alpha}}=\frac{\gamma-1}{2 \alpha} \lim _{\varepsilon \rightarrow 1-0} \frac{\varepsilon^{\gamma-2}}{(1-\varepsilon)^{2 \alpha-1}}=+\infty
$$

for every $\alpha>1 / 2$. From this we obtain desired result. The case $\gamma \leq 1$ is analogous.

\section{References}

[1] L. Bers, Mathematical Aspects of Subsonic and Transonic Gas Dynamics, Surveys in Applied Mathematics, vol. 3, John Wiley \& Sons; New York; Chapman \& Hall; London, 1958.

[2] M. A. Lavrentiev and B. V. Shabat, Problems of Hydrodynamics and Their Mathematical Models, Nauka, Moscow, 1973.

[3] G. Alessandrini and V. Nesi, Univalent $\sigma$-harmonic mappings, Archive for Rational Mechanics and Analysis 158 (2001), 155-171.

[4] D. Faraco, Beltrami operators and microstructure, Academic dissertation, Department of Mathematics, Faculty of Science, University of Helsinki, Helsinki, 2002.

[5] V. M. Miklyukov, On a new aproach to Bernshtein's theorem and related questions for equations of minimal surface type, Matematicheskii Sbornik. Novaya Seriya 108(150) (1979), no. 2, 268-289, 304, English translation in Mathematics of the USSR Sbornik 36 (1980), no. 2, 251-271.

[6] J. F. Hwang, Comparison principles and Liouville theorems for prescribed mean curvature equations in unbounded domains, Annali della Scuola Normale Superiore di Pisa 15 (1988), no. 3, 341355. 
[7] _ A uniqueness theorem for the minimal surface equation, Pacific Journal of Mathematics 176 (1996), no. 2, 357-364.

[8] P. Collin and R. Krust, Le probléme de Dirichlet pour l'equation des surfaces minimales sur des domaines non bornés, Bulletin de la Société Mathématique de France 119 (1991), no. 4, 443458.

[9] S. Pigola, M. Rigoli, and A. G. Setti, Some remarks on the prescribed mean curvature equation on complete manifolds, Pacific Journal of Mathematics 206 (2002), no. 1, 195-217.

V. A. Klyachin: Department of Mathematics, Volgograd State University,

Universitetsky Prospekt 100, 400062 Volgograd, Russia

E-mail address: klchnv@mail.ru

A. V. Kochetov: Department of Mathematics, Volgograd State University,

Universitetsky Prospekt 100, 400062 Volgograd, Russia

E-mail address: kochetov.alexey@mail.ru

V. M. Miklyukov: Department of Mathematics, Volgograd State University, Universitetsky Prospekt 100, 400062 Volgograd, Russia

E-mail address:miklyuk@mail.ru 